\title{
Engineering the thermotolerant industrial yeast Kluyveromyces marxianus for anaerobic growth ${ }^{\text {th }}$
}

\author{
Wijbrand J.C. Dekker, Raúl A. Ortiz-Merino, Astrid Kaljouw, Julius Battjes, Frank W. Wiering, \\ Christiaan Mooiman, Pilar de la Torre, Jack T. Pronk"
}

Department of Biotechnology, Delft University of Technology, van der Maasweg 9, 2629, HZ Delft, the Netherlands

\section{A R T I C L E I N F O}

\section{Keywords:}

Ergosterol

Tetrahymanol

Anaerobic metabolism

Thermotolerance

Ethanol production

Yeast biotechnology

Metabolic engineering

\begin{abstract}
A B S T R A C T
Current large-scale, anaerobic industrial processes for ethanol production from renewable carbohydrates predominantly rely on the mesophilic yeast Saccharomyces cerevisiae. Use of thermotolerant, facultatively fermentative yeasts such as Kluyveromyces marxianus could confer significant economic benefits. However, in contrast to $S$. cerevisiae, these yeasts cannot grow in the absence of oxygen. Responses of $K$. marxianus and $S$. cerevisiae to different oxygen-limitation regimes were analyzed in chemostats. Genome and transcriptome analysis, physiological responses to sterol supplementation and sterol-uptake measurements identified absence of a functional sterol-uptake mechanism as a key factor underlying the oxygen requirement of $K$. marxianus. Heterologous expression of a squalene-tetrahymanol cyclase enabled oxygen-independent synthesis of the sterol surrogate tetrahymanol in K. marxianus. After a brief adaptation under oxygen-limited conditions, tetrahymanol-expressing K. marxianus strains grew anaerobically on glucose at temperatures of up to $45{ }^{\circ} \mathrm{C}$. These results open up new directions in the development of thermotolerant yeast strains for anaerobic industrial applications.
\end{abstract}

\section{Introduction}

In terms of product volume (87 Mton $\mathrm{y}^{-1}$ ) (“Annual World Fuel Ethanol Production, 2020; Jansen et al., 2017), anaerobic conversion of carbohydrates into ethanol by the yeast Saccharomyces cerevisiae is the single largest process in industrial biotechnology. For fermentation products such as ethanol, anaerobic process conditions are required to maximize product yields and to minimize both cooling costs and complexity of bioreactors (Weusthuis et al., 2011). While $S$. cerevisiae is applied in many large-scale processes and is readily accessible to modern genome-editing techniques (Favaro et al., 2019; Stovicek et al., 2017), several non-Saccharomyces yeasts have traits that are attractive for industrial application. In particular, the high maximum growth temperature of thermotolerant yeasts, such as Kluyveromyces marxianus (up to $50{ }^{\circ} \mathrm{C}$ as opposed to $39{ }^{\circ} \mathrm{C}$ for $S$. cerevisiae), could enable lower cooling costs (Choudhary et al., 2016; Hong et al., 2007; Laman Trip and Youk, 2020). Moreover, it could reduce the required dosage of fungal polysaccharide hydrolases during simultaneous saccharification and fermentation (SSF) processes (Mejía-Barajas et al., 2018; Thorwall et al., 2020). However, as yet unidentified oxygen requirements hamper implementation of Kluyveromyces species in large-scale anaerobic processes (Kiers et al., 1998; Merico et al., 2007; Snoek and Steensma, 2006; Visser et al., 1990).

In $S$. cerevisiae, fast anaerobic growth on synthetic media requires supplementation with a source of unsaturated fatty acids (UFA), sterols, as well as several vitamins (Andreasen and Stier, 1953a, 1953b; Passi et al., 1993; Verduyn et al., 1990). These nutritional requirements reflect well-characterized, oxygen-dependent biosynthetic reactions. UFA synthesis involves the oxygen-dependent acyl-CoA desaturase Ole1, $\mathrm{NAD}^{+}$synthesis depends on the oxygenases Bna2, Bna4, and Bna1, while de novo synthesis of ergosterol, the main yeast sterol, even requires 12 mol of oxygen per mole.

Oxygen-dependent reactions in $\mathrm{NAD}^{+}$synthesis can be bypassed by nutritional supplementation of nicotinic acid, which is a standard ingredient of synthetic media for cultivation of $S$. cerevisiae (Perli et al., 2020; Verduyn et al., 1990). Ergosterol and the UFA source Tween 80 (polyethoxylated sorbitan oleate) are routinely included in media for anaerobic cultivation as 'anaerobic growth factors' (AGF) (Andreasen and Stier, 1953b; Dekker et al., 2019; Verduyn et al., 1990). Under anaerobic conditions, $S$. cerevisiae imports exogenous sterols via the ABC

\footnotetext{
Manuscript for submission in Metabolic Engineering, section: Article.

* Corresponding author.

E-mail address: j.t.pronk@tudelft.nl (J.T. Pronk).
} 
transporters Aus1 and Pdr11 (Wilcox et al., 2002). Mechanisms for uptake and hydrolysis of Tween 80 by $S$. cerevisiae are unknown but, after its release, oleate is activated by the acyl-CoA synthetases Faa1 and Faa4 (Black and DiRusso, 2007; Jacquier and Schneiter, 2010).

Outside the whole-genome duplicated (WGD) clade of Saccharomycotina yeasts, only few yeasts (including Candida albicans and Brettanomyces bruxellensis) are capable of anaerobic growth in synthetic media supplemented with vitamins, ergosterol and Tween 80 (Blomqvist et al., 2012; Merico et al., 2007; Visser et al., 1990; Zavrel et al., 2013). However, most currently known yeast species readily ferment glucose to ethanol and carbon dioxide when exposed to oxygen-limited growth conditions (Dashko et al., 2014; Merico et al., 2007; Visser et al., 1990), indicating that they do not depend on respiration for energy conservation. The inability of the large majority of facultatively fermentative yeast species to grow under strictly anaerobic conditions is therefore commonly attributed to incompletely understood oxygen requirements for biosynthetic processes (Snoek and Steensma, 2006). Several oxygen-requiring processes have been proposed, including involvement of a respiration-coupled dihydroorotate dehydrogenase in pyrimidine biosynthesis, limitations in uptake and/or metabolism of anaerobic growth factors, and redox-cofactor balancing constraints (Merico et al., 2007; Snoek and Steensma, 2006, 2007).

Quantitation, identification and elimination of oxygen requirements in non-Saccharomyces yeasts is hampered by the very small amounts of oxygen required for non-dissimilatory purposes. For example, preventing entry of the small amounts of oxygen required for sterol and UFA synthesis in laboratory-scale bioreactor cultures of $S$. cerevisiae requires extreme measures, such as used for anaerobic workstations, leading ultra-pure nitrogen gas over cultures, use of bioreactor tubing, seals and sensors that are resistant to oxygen diffusion and prevention of 'carryover' of anaerobic growth factors from aerobically grown inocula (da Costa et al., 2018; Mooiman et al., 2021; Visser et al., 1990). These technical challenges are likely to have contributed to reports on anaerobic growth of several non-Saccharomyces yeasts. In addition, the term 'anaerobic' is often applied to cultivation systems in which the contribution of respiration to energy metabolism is quantitatively insignificant relative to that of fermentation. For example, several publications that report anaerobic growth of $K$. marxianus do not detail key measures required to minimize entry of the small amounts of oxygen required for biosynthetic processes (Hughes et al., 2013; Tetsuya et al., 2013; Wilkins et al., 2008). Paradoxically, the same small oxygen requirements that, without special measures, go unnoticed in laboratory cultures can represent a real challenge in large-scale bioreactors, in which oxygen availability is limited by low surface-to-volume ratios and vigorous carbon-dioxide production.

Identification of the non-dissimilatory oxygen requirements of nonconventional yeast species is required to eliminate a key bottleneck for their application in industrial anaerobic processes and, on a fundamental level, can shed light on the roles of oxygen in eukaryotic metabolism. The goal of this study was to identify and eliminate the nondissimilatory oxygen requirements of the facultatively fermentative, thermotolerant yeast $K$. marxianus. To this end, we analyzed and compared physiological and transcriptional responses of $K$. marxianus and $S$. cerevisiae to different oxygen- and anaerobic-growth factor limitation regimes in chemostat cultures. Based on the outcome of this comparative analysis, subsequent experiments focused on characterization and engineering of sterol metabolism and yielded $K$. marxianus strains that grew anaerobically at $45^{\circ} \mathrm{C}$.

\section{Results}

\subsection{K. marxianus and S. cerevisiae show different physiological responses to oxygen limitation}

In ideally mixed, steady-state chemostat cultures, the applied dilution rate $\left(D, h^{-1}\right)$ equals the specific growth rate. Chemostat cultivation therefore enables analysis of the impact of cultivation conditions on microbial physiology and genome expression at a fixed specific growth rate (Daran-Lapujade et al., 2008). To investigate oxygen requirements of $K$. marxianus, physiological responses of strain CBS6556 to different oxygenation regimes and anaerobic growth-factor (AGF) limitation regimes (Fig. 1a) were studied in glucose-grown chemostat cultures. The chemostats were operated at a $D$ of $0.10 \mathrm{~h}^{-1}$, which is used as a reference value in many yeast physiology studies (Tai et al., 2005; Van Eunen et al., 2010). Physiological parameters of $K$. marxianus in these cultures were compared to those of $S$. cerevisiae CEN.PK113-7D subjected to the same cultivation regimes.

When glucose-limited, aerobic chemostat cultures with a working volume of $1.2 \mathrm{~L}$ were supplied with $0.5 \mathrm{~L}$ air. $\mathrm{min}^{-1}\left(21 \cdot 10^{4} \mathrm{ppm} \mathrm{O}_{2}\right.$ in inlet gas flow, corresponding to $258 \mathrm{mmol} \mathrm{O}_{2} \mathrm{~h}^{-1}$, Condition 1), the Crabtree-negative yeast $K$. marxianus (van Urk et al., 1989) and the Crabtree-positive yeast S. cerevisiae (von Meyenburg, 1969) both exhibited a fully respiratory dissimilation of glucose, as evident from absence of ethanol production and a respiratory quotient (RQ) close to 1 (Table 1). Apparent biomass yields on glucose of both yeasts exceeded $0.5 \mathrm{~g}$ biomass (g glucose) ${ }^{-1}$ and were approximately $10 \%$ higher than previously reported due to co-consumption of ethanol, which was used as solvent for the anaerobic growth factor ergosterol (Rouwenhorst et al., 1988; van Urk et al., 1989).

When the oxygen content in the inlet gas was reduced to $840 \mathrm{ppm}$ (2.1 $\mathrm{mmol} \mathrm{O}_{2} \mathrm{~h}^{-1}$, Condition 2), both yeasts showed a much lower biomass-specific respiration rate $\left(\mathrm{q}_{\mathrm{O} 2}\right)$ than in the aerobic cultures (Fig. 1b), resulting in a mixed respiro-fermentative glucose metabolism. RQ values close to 50 and biomass-specific ethanol-production rates of $11.5 \pm 0.6 \mathrm{mmol} \mathrm{g}{ }^{-1} \cdot \mathrm{h}^{-1}$ for $K$. marxianus and $7.5 \pm 0.1 \mathrm{mmol} \mathrm{g}{ }^{-1} \cdot \mathrm{h}^{-1}$ for $S$. cerevisiae (Table 1), indicated that glucose dissimilation in Condition 2 was predominantly fermentative. In these oxygen-limited cultures, a higher concentration of glucose $\left(20 \mathrm{~g} \mathrm{~L}^{-1}\right)$ was included in the medium feed than in the aerobic cultures $\left(7.5 \mathrm{~g} \mathrm{~L}^{-1}\right)$ to compensative for the lower biomass yield of oxygen-limited, predominantly fermentative yeast cultures (Kiers et al., 1998; Weusthuis et al., 1994). Biomass-specific rates of glycerol production which, under oxygen-limited conditions is responsible for re-oxidation of NADH generated in biosynthetic reactions (Bakker et al., 2001), were approximately 2.5 -fold higher $\left(p=2.3 \cdot 10^{-4}\right)$ in $K$. marxianus than in $S$. cerevisiae. Glycerol production further confirmed that, for both yeasts, the reduced oxygen-supply rate in Condition 2 constrained mitochondrial respiration (Weusthuis et al., 1994). However, low residual glucose concentrations (Fig. 1, Table 1) indicated that sufficient oxygen was provided to meet most or all of the biosynthetic oxygen requirements of K. marxianus.

To explore growth under an even more stringent oxygen-limitation regime, we exploited previously documented challenges in achieving complete anaerobiosis in laboratory bioreactors (da Costa et al., 2018; Dekker et al., 2019; Mooiman et al., 2021). Even in chemostats sparged with pure nitrogen, which contained $<0.5$ ppm oxygen $(<0.13 \mu \mathrm{mol}$ $\mathrm{min}^{-1}$, Conditions 3,4 and 5), S. cerevisiae grew on synthetic medium lacking Tween 80 and ergosterol, albeit at an increased residual glucose concentration (Condition 5, Fig. 1, Table 1). In contrast, $K$. marxianus cultures sparged with pure $\mathrm{N}_{2}$ and supplemented with both AGFs consumed only $20 \%$ of the glucose fed to the cultures (Condition 3 ). These severely oxygen-limited cultures showed a high residual glucose concentration of $15.9 \pm 0.3 \mathrm{~g} \mathrm{~L}^{-1}$ and a low but constant biomass concentration of $0.4 \pm 0.0 \mathrm{~g} \mathrm{~L}^{-1}$ (Fig. 1B, Table 1). This pronounced response of $K$. marxianus to extreme oxygen limitation provided an experimental context for further analyzing its unknown oxygen requirements.

$S$. cerevisiae can import exogenous sterols under severely oxygenlimited or anaerobic conditions (Wilcox et al., 2002). If the latter were also true for K. marxianus, omission of ergosterol from the growth medium of severely oxygen-limited cultures would increase biomass-specific oxygen requirements and lead to an even lower 
a

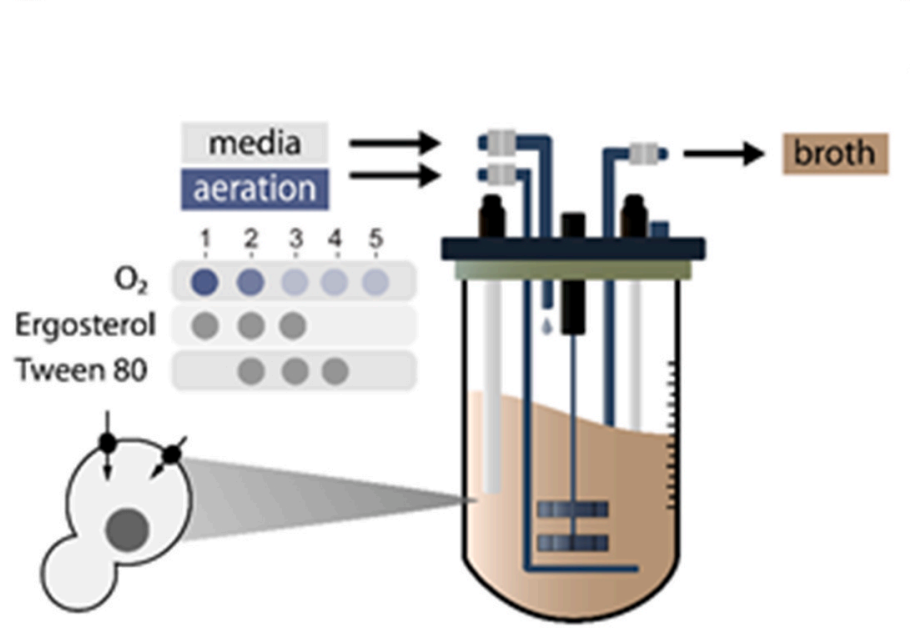

b S. cerevisiae

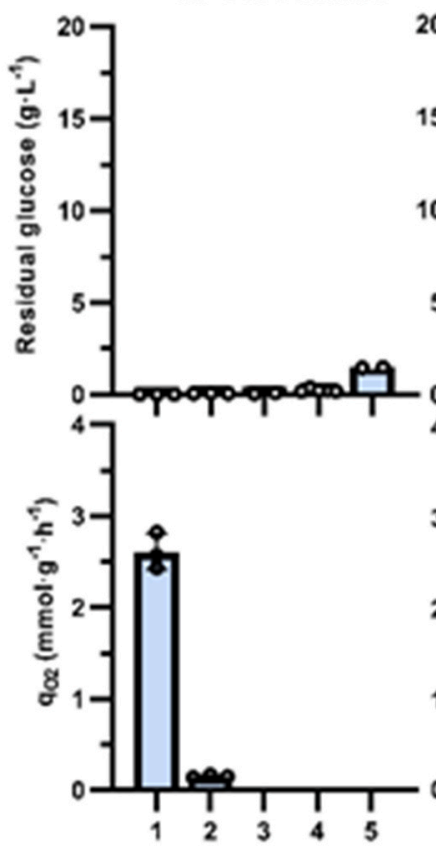

K. marxianus

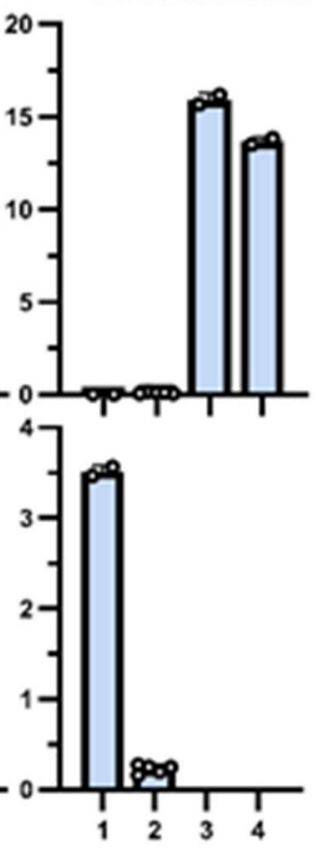

\begin{tabular}{|c|c|c|c|c|}
\hline Condition & $\mathrm{ppm}$ & AGF & Sc & $\mathrm{Km}$ \\
\hline 1 & $21 \cdot 10^{4}$ & $E$ & 1.00 & 1.00 \\
\hline 2 & 840 & $\mathrm{TE}$ & 1.00 & 1.00 \\
\hline 3 & $<0.5$ & TE & 1.00 & 1.00 \\
\hline 4 & $<0.5$ & $T$ & 1.00 & 1.00 \\
\hline 5 & $<0.5$ & - & 1.00 & - \\
\hline
\end{tabular}

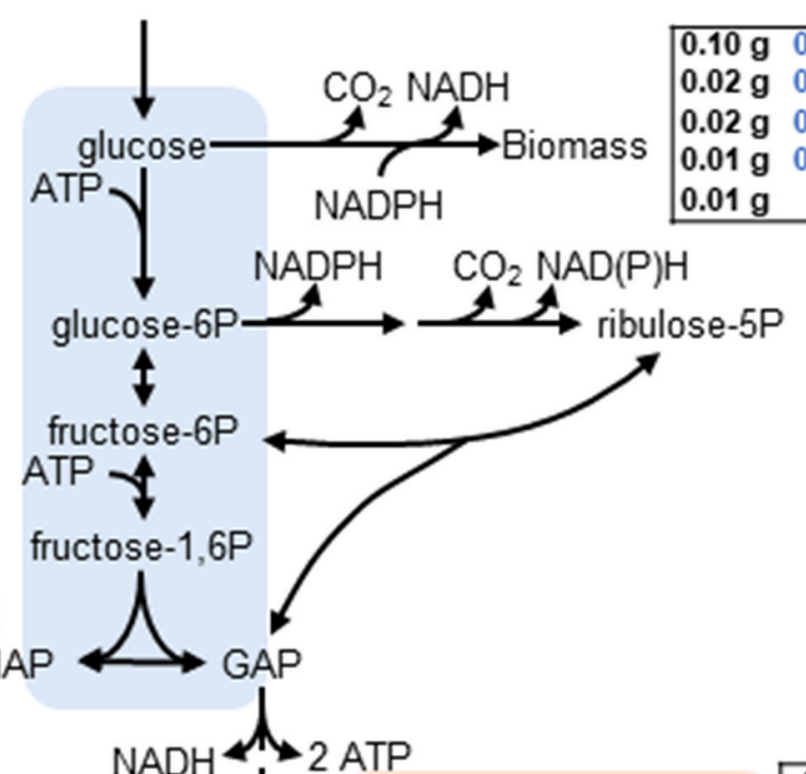

\begin{tabular}{|ll|}
\hline $\mathbf{0 . 0 0}$ & 0.00 \\
$\mathbf{0 . 1 0}$ & 0.15 \\
$\mathbf{0 . 1 3}$ & 0.27 \\
$\mathbf{0 . 1 2}$ & 0.25 \\
$\mathbf{0 . 1 5}$ & - \\
\hline
\end{tabular}

glycerol $\longleftarrow \leftarrow$ DHAP

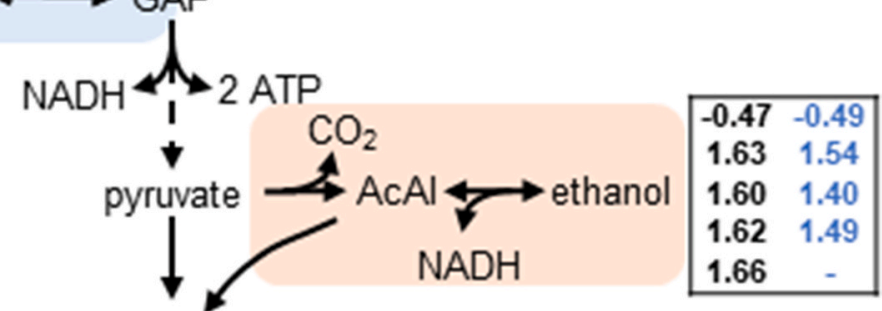

TCA cycle

(mitochondria)

Fig. 1. Chemostat cultivation of $S$. cerevisiae CEN.PK113-7D and $K$. marxianus CBS6556 under different oxygen-supply and anaerobic-growth-factor (AGF) supplementation regimes. Chemostat cultures were grown on SMG-urea, at pH 6.0 , at $30{ }^{\circ} \mathrm{C}$ and at a dilution rate of $0.10 \mathrm{~h}^{-1}$. The ingoing gas flow of $500 \mathrm{~mL}$ min ${ }^{-1}$ contained either $21 \cdot 10^{4} \mathrm{ppm} \mathrm{O}_{2}$ (Condition 1), $840 \mathrm{ppm} \mathrm{O}_{2}$ (condition 2), or $<0.5 \mathrm{ppm} \mathrm{O}$ (conditions 3,4 and 5). Cultures were grown with or without supplementation ergosterol (E) and/or Tween 80 (T). a, Schematic representation of experimental set-up and growth conditions. Grey dots indicate cultures supplemented with ergosterol and/or Tween 80 and intensity of the blue dots represents oxygen supply to the cultures. Data for each condition were obtained from independent replicate chemostat cultures. $\mathbf{b}$, Residual glucose concentrations and biomass-specific oxygen-consumption rates ( $\mathrm{q}_{\mathrm{O} 2}$ ) in chemostat cultures grown under the different oxygen-supply and AGF-supplementation regimes. Data represent mean and standard deviation of independent replicate chemostat cultures. c, Distribution of consumed glucose over biomass and products in chemostat cultures of $S$. cerevisiae (left column) and $K$. marxianus (right column), normalized to a glucose uptake rate of $1.00 \mathrm{~mol} \mathrm{~h}^{-1}$. Numbers in boxes indicate averages of measured metabolite formation rates $\left(\mathrm{mol} \cdot \mathrm{h}^{-1}\right.$ ) and biomass production rates $(\mathrm{g}$ dry weight $\cdot h^{-1}$ ) for each condition. 
Table 1

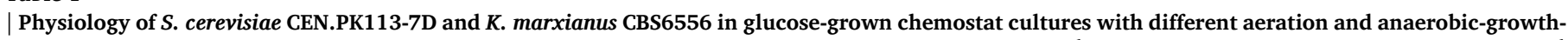

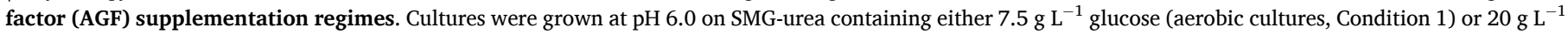

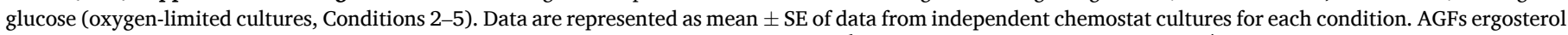

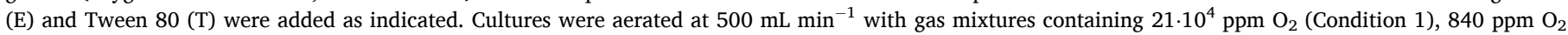

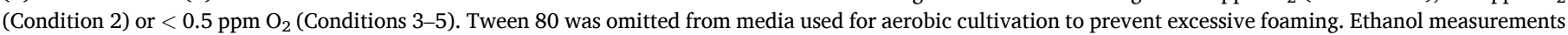

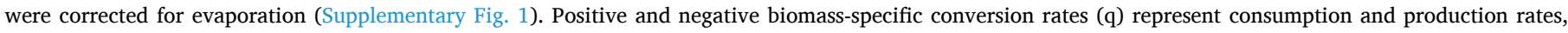
respectively. Respiratory quotient (RQ) is equal to $\mathrm{qCO}_{2} / \mathrm{qO}_{2}$ ).

\begin{tabular}{|c|c|c|c|c|c|c|c|c|c|}
\hline \multirow{2}{*}{$\frac{\text { Yeast strain }}{\text { Condition }}$} & \multicolumn{5}{|c|}{ S. cerevisiae CEN.PK113-7D } & \multicolumn{4}{|c|}{ K. marxianus CBS6556 } \\
\hline & 1 & 2 & 3 & 4 & 5 & 1 & 2 & 3 & 4 \\
\hline $\mathrm{O}_{2}$ in inlet gas (ppm) & $21 \cdot 10^{4}$ & 840 & 0.5 & 0.5 & 0.5 & $21 \cdot 10^{4}$ & 840 & 0.5 & 0.5 \\
\hline AGF & E & $\mathrm{TE}$ & $\mathrm{TE}$ & $\mathrm{T}$ & - & $\mathrm{E}$ & $\mathrm{TE}$ & $\mathrm{TE}$ & $\mathrm{T}$ \\
\hline Replicates & 3 & 3 & 2 & 5 & 2 & 2 & 5 & 2 & 2 \\
\hline Biomass $\left(\mathrm{g} \cdot \mathrm{L}^{-1}\right)$ & $4.22 \pm 0.06$ & $2.29 \pm 0.04$ & $1.98 \pm 0.01$ & $1.56 \pm 0.03$ & $1.12 \pm 0.02$ & $3.79 \pm 0.02$ & $1.57 \pm 0.10$ & $0.35 \pm 0.02$ & $0.50 \pm 0.04$ \\
\hline Residual glucose $\left(\mathrm{g} \cdot \mathrm{L}^{-1}\right)$ & $0.00 \pm 0.00$ & $0.07 \pm 0.00$ & $0.06 \pm 0.02$ & $0.23 \pm 0.04$ & $1.47 \pm 0.01$ & $0.00 \pm 0.00$ & $0.10 \pm 0.02$ & $16.0 \pm 0.3$ & $13.7 \pm 0.3$ \\
\hline \multicolumn{10}{|l|}{ Biomass-specific rates } \\
\hline Specific growth rate $\left(\mathrm{h}^{-1}\right)$ & $0.10 \pm 0.00$ & $0.10 \pm 0.00$ & $0.10 \pm 0.00$ & $0.10 \pm 0.00$ & $0.10 \pm 0.00$ & $0.10 \pm 0.00$ & $0.11 \pm 0.01$ & $0.12 \pm 0.01$ & $0.12 \pm 0.01$ \\
\hline $\mathrm{q}_{\text {glucose }}\left(\mathrm{mmol} \cdot \mathrm{g}^{-1} \cdot \mathrm{h}^{-1}\right)$ & $\begin{array}{l}-0.95 \pm \\
0.03\end{array}$ & $\begin{array}{l}-4.59 \pm \\
0.10\end{array}$ & $\begin{array}{l}-5.25 \pm \\
0.04\end{array}$ & $\begin{array}{l}-6.77 \pm \\
0.27\end{array}$ & $\begin{array}{l}-9.06 \pm \\
0.15\end{array}$ & $\begin{array}{l}-1.05 \pm \\
0.00\end{array}$ & $\begin{array}{l}-7.46 \pm \\
0.30\end{array}$ & $\begin{array}{l}-7.30 \pm \\
0.81\end{array}$ & $\begin{array}{l}-8.53 \pm \\
0.00\end{array}$ \\
\hline $\mathrm{q}_{\text {ethanol }}\left(\mathrm{mmol} \cdot \mathrm{g}^{-1} \cdot \mathrm{h}^{-1}\right)$ & $\begin{array}{l}-0.44 \pm \\
0.03\end{array}$ & $7.48 \pm 0.10$ & $8.40 \pm 0.02$ & $11.0 \pm 0.6$ & $15.0 \pm 0.5$ & $\begin{array}{l}-0.52 \pm \\
0.00\end{array}$ & $11.5 \pm 0.44$ & $10.3 \pm 0.7$ & $12.7 \pm 0.1$ \\
\hline $\begin{array}{l}\mathrm{q}_{\text {succinate }}\left(\mathrm{mmol} \cdot \mathrm{g}^{-1} \cdot \mathrm{h}^{-1}\right) \\
\text { Stoichiometries }\end{array}$ & $0.00 \pm 0.00$ & $0.00 \pm 0.00$ & $0.03 \pm 0.00$ & $0.03 \pm 0.01$ & $0.06 \pm 0.00$ & $0.00 \pm 0.00$ & $0.02 \pm 0.00$ & $0.01 \pm 0.01$ & $0.04 \pm 0.00$ \\
\hline RQ & $1.08 \pm 0.02$ & $52.2 \pm 2.4$ & - & - & - & $1.06 \pm 0.01$ & $49.3 \pm 7.5$ & - & - \\
\hline $\begin{array}{l}\text { Y glycerol/biomass } \\
\left(\mathrm{mmol} \cdot \mathrm{g}^{-1}\right)\end{array}$ & $0.00 \pm 0.00$ & $3.67 \pm 0.05$ & $5.58 \pm 0.02$ & $6.73 \pm 0.25$ & $11.3 \pm 0.4$ & $0.00 \pm 0.00$ & $9.51 \pm 0.46$ & $16.9 \pm 0.8$ & $18.5 \pm 2.1$ \\
\hline $\mathrm{Y}$ biomass/glucose $\left(\mathrm{g} \cdot \mathrm{g}^{-1}\right)$ & $0.57 \pm 0.01$ & $0.12 \pm 0.00$ & $0.10 \pm 0.00$ & $0.08 \pm 0.00$ & $0.06 \pm 0.00$ & $0.53 \pm 0.00$ & $0.08 \pm 0.00$ & $0.09 \pm 0.00$ & $0.09 \pm 0.01$ \\
\hline Y ethanol/glucose $\left(g \cdot g^{-1}\right)$ & - & $1.67 \pm 0.06$ & $1.63 \pm 0.02$ & $1.65 \pm 0.02$ & $1.68 \pm 0.02$ & - & $1.53 \pm 0.03$ & $1.31 \pm 0.05$ & $1.40 \pm 0.02$ \\
\hline \multicolumn{10}{|l|}{ Recoveries (out/in) } \\
\hline Carbon (\%) & $99.9 \pm 0.7$ & $101.2 \pm 3.3$ & $100.4 \pm 0.1$ & $100.1 \pm 1.3$ & $104.0 \pm 0.2$ & $100.5 \pm 0.1$ & $91.1 \pm 2.0$ & $101.6 \pm 6.5$ & $99.7 \pm 3.9$ \\
\hline Degree of reduction (\%) & $98.4 \pm 0.7$ & $100.9 \pm 0.8$ & $100.1 \pm 0.9$ & $98.1 \pm 0.6$ & $100.1 \pm 1.8$ & $98.8 \pm 0.1$ & $94.5 \pm 0.4$ & $97.8 \pm 6.2$ & $99.1 \pm 3.5$ \\
\hline
\end{tabular}

biomass concentration. In practice however, omission of ergosterol from the medium feed of severely oxygen-limited chemostat cultures (Condition 4, Fig. 1b, Table 1) led to a small increase of the biomass concentration and a corresponding decrease of the residual glucose concentration relative to severely oxygen-limited cultures that were supplemented with ergosterol (Condition 3 ). This observation suggested that, in contrast to $S$. cerevisiae, $K$. marxianus cannot replace de novo oxygen-dependent synthesis of sterols by uptake of exogenous ergosterol.

\subsection{Transcriptional responses of $K$. marxianus to oxygen limitation involve ergosterol metabolism}

To further investigate the inability of $K$. marxianus to grow anaerobically, transcriptome analyses were performed on chemostat cultures of $S$. cerevisiae and $K$. marxianus. A genome sequence of $K$. marxianus CBS6556 was only available as draft, non-annotated assembly (Jeong et al., 2012). Therefore, long-read genome sequencing, assembly and de novo genome annotation were performed and the annotation was refined by using transcriptome assemblies (see Data availability). Complete transcriptome datasets for the tested combinations of growth conditions and yeast strains shown in Fig. 1 are available (see Data availability).

Analysis of the transcriptome data was focused on orthologous genes in $S$. cerevisiae and $K$. marxianus under a subset of the tested conditions (Figs. 2 and 3). Contrast 31, which captured a comparison between conditions 3 ( $<0.5 \mathrm{ppm} \mathrm{O}_{2}$ in inlet gas, severely oxygen-limited, supplemented with Tween 80 and ergosterol) and 1 (aerobic, glucoselimited), revealed large differences between $S$. cerevisiae and K. marxianus (Fig. 2). Of the $3 \mathrm{GO}$ categories (biological processes) that were enriched among $S$. cerevisiae genes upregulated under severe oxygen limitation (Condition 3), only one (rRNA processing) was also found in $K$. marxianus with the same directionality change. Likewise, 12 GO categories that were overrepresented among genes that were downregulated in this contrast in $S$. cerevisiae cultures, only 7 were similarly overrepresented among down-regulated genes in $K$. marxianus. A comparison of contrast 31 for all orthologous genes in $S$. cerevisiae and
K. marxianus also revealed large differences (Fig. 2b).

Although low, residual glucose concentrations in the severely oxygen-limited (Condition 3) S. cerevisiae cultures were significantly higher than in aerobic, glucose-limited cultures (Condition 1, Table 1). Closer inspection of the enriched GO categories (Fig. 2B) for contrast 31 revealed GO categories that, in $S$. cerevisiae, harbor many genes whose transcription is regulated by glucose. For example, many $S$. cerevisiae genes involved in carnitine metabolism, TCA cycle and glyoxylate cycle are all transcriptionally repressed by glucose (Schüller, 2003). The much higher glucose concentration in the severely oxygen-limited K. marxianus cultures (Fig. 1, Table 1) may therefore have exerted an even stronger impact on contrast 31 for this yeast. In an attempt to exclude genes for which, in $S$. cerevisiae, glucose regulation masks a transcriptional response to oxygen availability, contrast 31 was studied for a subset of $S$. cerevisiae genes that show consistent transcriptional upor down-regulation in anaerobic chemostat cultures grown under different nutrient limitations (Tai et al., 2005). Also for this subset, transcriptional responses of the corresponding K. marxianus orthologs to severe oxygen limitation were mostly different from those in $S$. cerevisiae (Fig. 2bc). This observation suggested that not all differences observed for contrast 31 can be attributed to different glucose concentrations and that, instead, transcriptional responses to oxygen availability of $S$. cerevisiae and $K$. marxianus are substantially different.

K. marxianus harbors two dihydroorotate dehydrogenases, a cytosolic fumarate-dependent enzyme (KmUra1) and a mitochondrial quinone-dependent enzyme (KmUra9). In vivo activity of the latter requires oxygen because the reduced quinone is reoxidized by the mitochondrial respiratory chain (Nagy et al., 1992). Consistent with the different oxygen requirements of pyrimidine synthesis via KmUra9 and ScUra1, KmURA9 showed lower transcript levels under severely oxygen-limited conditions than in aerobic, glucose-limited cultures, while KmURA1 was upregulated under severe oxygen limitation (Fig. 2b, contrast 31). Upregulation of KmURA1 coincided with increased production of succinate (Table 1 ).

To test our hypothesis that absence of a functional sterol uptake system plays a key role in the inability of $K$. marxianus to grow 
a

GO:0006096 glycolytic process GO:0006696 ergosterol

(1) Go.00060 Go.0. Golosynthetic Go 00192 isopentenyl GO: 0015680 protein maturation by GO:0009396 folic acid-containing GO.004420 (1)

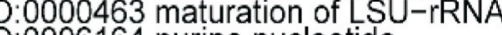
GO:0006164 purine nucleotide SSU-rRNA Go.000447 endonucleolytic GO:0000480 end nucleolytic GO 0008652 cellular amino acid GO 0006364 rRNA processing G006629 lipid metabolic O 006094 lud o:000 GO:0 GO 0000302 response to reactive GO:0042744 hyonen peroxide GO 0008152 metabolic process GO:0006072 glycerol-3-phosphate GO:0055114 oxidation-reduction GO:0006116 NADH oxidation GO.0006631 fatty acid metaboli GO:0055085 transmembrane transport GO:0006754 ATP biosynthetic process GO:0009060 aerobic respiration GO:0006811 ion transport GO:0005975 carbohydrate metabolic GO:0006121 mitochondrial electron GO:1902600 proton transmembrane GO:0006099 tricarboxylic acid cycle GO:0006635 fatty acid

GO:0006123 mitochondrial electron GO:0006122 mitochondrial electron GO:0019629 propionate catabolic GO:0006119 oxidative GO:0015986 ATP synthesis coupled GO:0006102 isocitrate metabolic GO:0035948 obsolete positive GO:0061414 positive regulation of GO:0006012 galactose metabolic

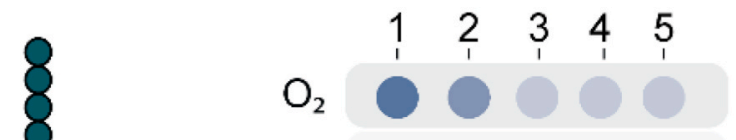

Ergosterol

Tween 80

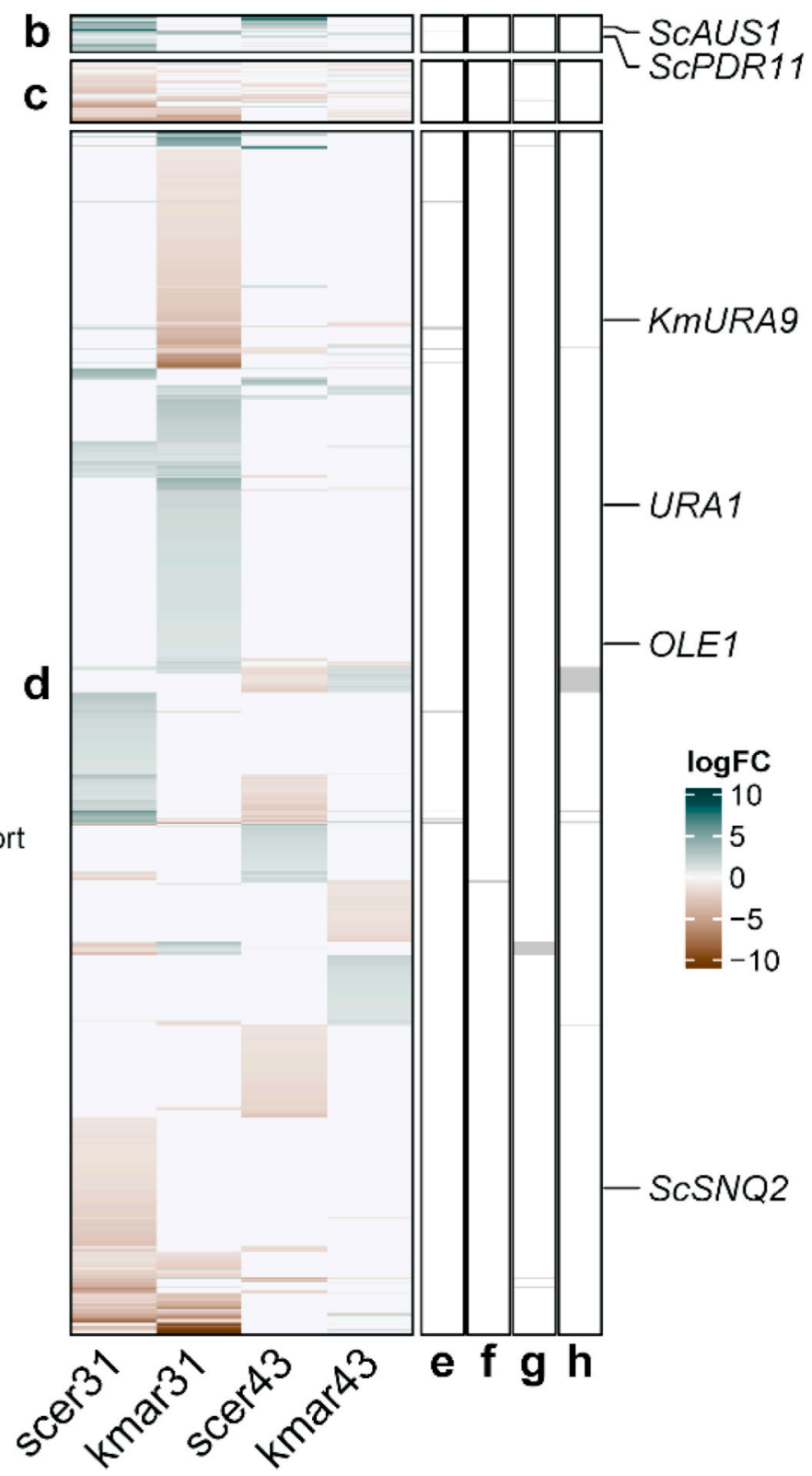

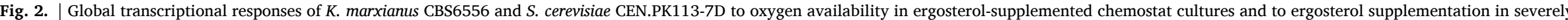

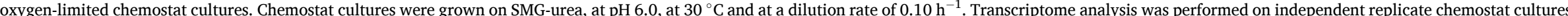

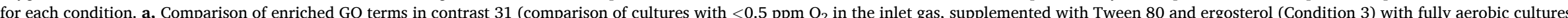

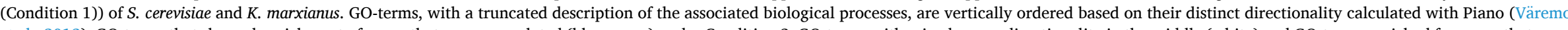

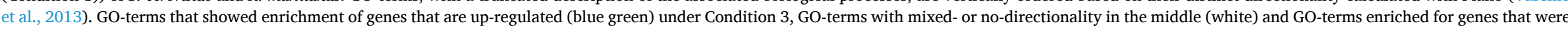

GO:0006950 response to stress Gotabolic GO:0000023 maltose metabolic GO:0030476 ascospore wall assen GO:0006116 NADH oxidation GO:0006846 acetate transport GO:0015744 succinate transport GO:0015741 fumarate transport GO:0019740 nitrogen utilization Go:0015696 ammonium transport GO:0055085 transmembrane transport Go:0055114 oxidation-reduction GO:0008152 metabolic process G:0006811 ion transport GO:0006631 glyoxylate cycle GO:0006629 lipid metabolic process GO:0006099 tricarboxylic acid cycle GO:0006810 transport GO:0006635 fatty acid G:0009437 carnitine metabolic GO:0006833 water transport

cer 


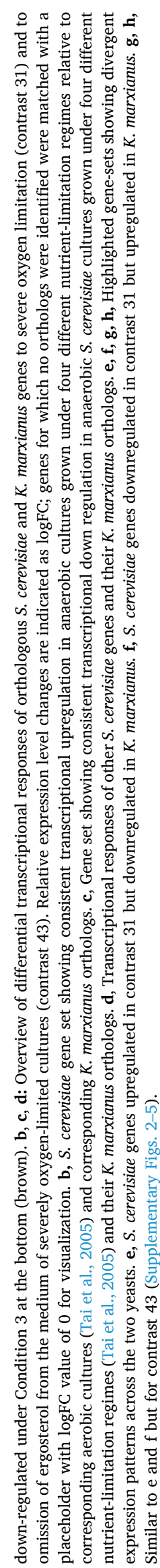

anaerobically, further analysis of the transcriptome data was focused on expression of genes involved in sterol metabolism. In $S$. cerevisiae, import of exogenous sterols by Aus1 and Pdr11 can alleviate the impact of oxygen limitation on sterol biosynthesis (Wilcox et al., 2002). Consistent with this role of sterol uptake, sterol biosynthetic genes in $S$. cerevisiae were highly upregulated in severely oxygen-limited cultures when ergosterol in contrast 43 , which captured the transcriptional response of oxygen-limited cultures upon omission of ergosterol from the growth medium (Fig. 3b, Supplementary Fig. 6). Also the mevalonate pathway for synthesis of the sterol precursor squalene, which does not require oxygen, was upregulated in this comparison, consistent with a relief of feedback regulation by ergosterol (Jordá and Puig, 2020). In contrast to $S$. cerevisiae, $K$. marxianus showed a higher transcript levels of genes involved in sterol, isoprenoid and fatty-acid metabolism in severely oxygen-limited cultures supplemented with ergosterol and Tween 80 than in aerobic, glucose-limited cultures (Fig. 2ab, Fig. 3, contrast 31). No further increase of the expression levels of sterol-biosynthesis genes was observed in the latter yeast upon omission of ergosterol from the medium of severely oxygen-limited cultures (Supplementary Fig. 6, contrast 43). These results were consistent with our hypothesis that, in contrast to $S$. cerevisiae, $K$. marxianus is unable to import ergosterol when sterol synthesis is compromised. Moreover, co-orthology prediction with Proteinortho (Lechner et al., 2011) revealed no orthologs of the $S$. cerevisiae sterol transporters Aus1 and Pdr11 in K. marxianus.

\subsection{Absence of sterol import in K. marxianus}

To test the hypothesis that $K$. marxianus lacks a functional steroluptake mechanism, uptake of fluorescent sterol derivative 25-NBDcholesterol (NBDC) was measured by flow cytometry (Marek et al., 2014). Since $S$. cerevisiae sterol transporters are not expressed in aerobic conditions (Wilcox et al., 2002) and to avoid interference of sterol synthesis, NBDC uptake was analyzed in anaerobic cell suspensions (Fig. 4a). Four hours after NBDC addition to cell suspensions of the reference strain $S$. cerevisiae IMX585, median single-cell fluorescence increased by 66-fold (Fig. 4bc). In contrast, the congenic sterol-transporter-deficient strain IMK809 (aus1 $\Delta$ pdr11 1 ) only showed a 6-fold increase of fluorescence, probably reflected detergent-resistant binding of NBDC to $S$. cerevisiae cell-wall proteins (Marek et al, 2011, 2014). K. marxianus strains CBS6556 and NBRC1777 did not show increased fluorescence, neither after $4 \mathrm{~h}$ nor after $23 \mathrm{~h}$ of incubation with NBDC ( $<2$-fold, Fig. 4bc, Supplementary Fig. 7).

\subsection{Engineering K. marxianus for oxygen-independent growth}

The mechanism of sterol uptake by $S$. cerevisiae, which in addition to a functional plasma-membrane transporter also requires cell wall proteins, has not yet been fully resolved (Alimardani et al., 2004; Marek et al., 2014). Instead of expressing a heterologous sterol-import system in $K$. marxianus, we therefore explored production of tetrahymanol, which acts as a sterol surrogate in strictly anaerobic fungi (Takishita et al., 2012). Expression of a squalene-tetrahymanol cyclase from Tetrahymena thermophila (TtSTC1), which catalyzes the single-step oxygen-independent conversion of squalene into tetrahymanol (Fig. 5a), was recently shown to enable sterol-independent growth of $S$. cerevisiae (Wiersma et al., 2020).

TtSTC1 was expressed in K. marxianus NBRC1777, which is more genetically amenable than strain CBS6556 (Rajkumar et al., 2019). After $40 \mathrm{~h}$ of anaerobic incubation, the resulting strain contained $2.4 \pm 0.4$ $\mathrm{mg} \cdot(\mathrm{g} \text { biomass })^{-1}$ tetrahymanol, $0.4 \pm 0.1 \mathrm{mg} \mathrm{g}^{-1}$ ergosterol and no detectable squalene, while strain NBRC1777 contained $3.5 \pm 0.1 \mathrm{mg} \mathrm{g}^{-1}$ squalene and $3.4 \pm 0.2 \mathrm{mg} \mathrm{g}^{-1}$ ergosterol (Fig. 5b). In strictly anaerobic cultures on sterol-free medium, strain NBRC1777 grew immediately after inoculation but not after transfer to a second anaerobic culture (Fig. 5c), consistent with 'carry-over' of ergosterol from the aerobic 
a

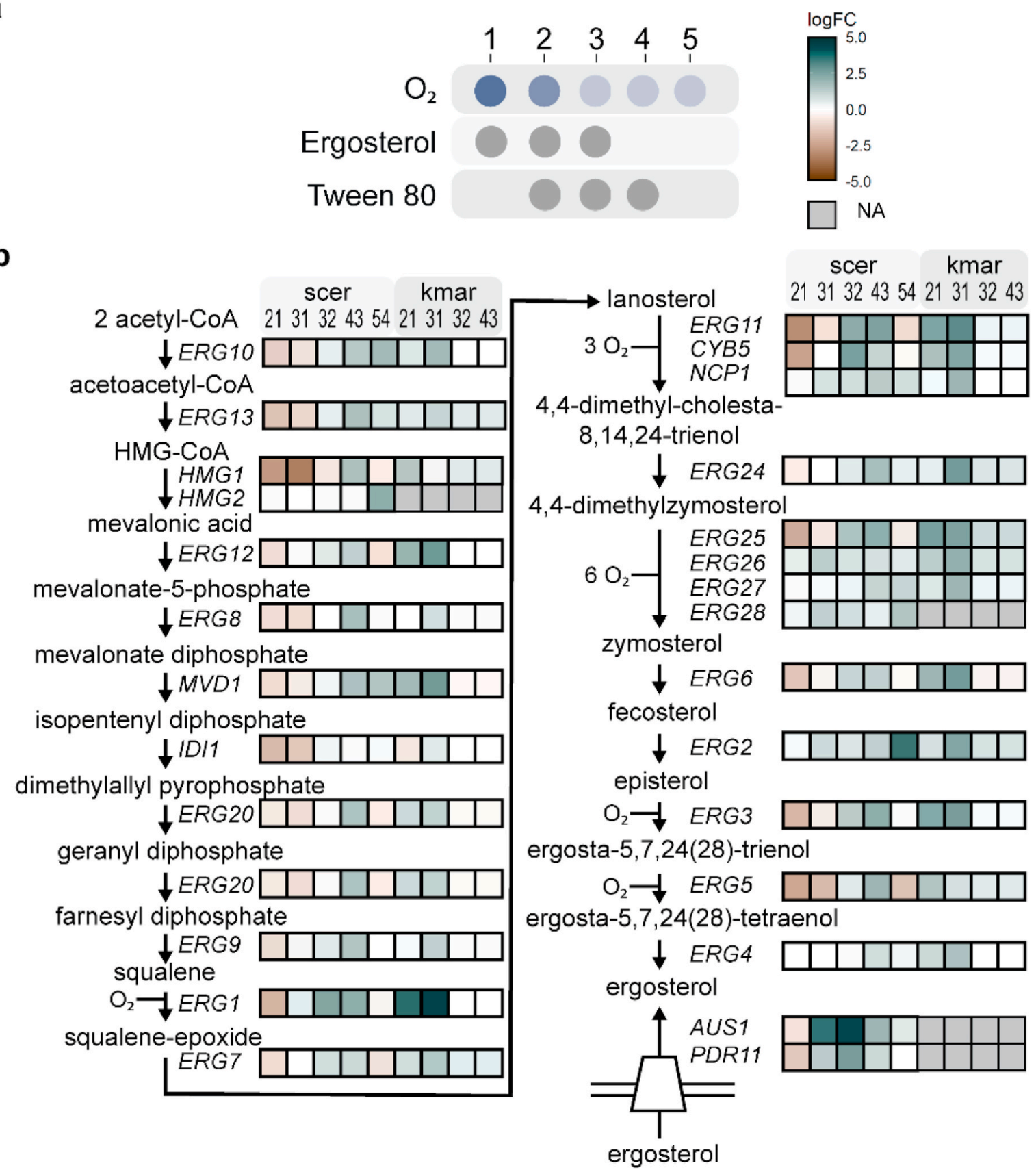

b

Fig. 3. | Different transcriptional regulation of ergosterol-biosynthesis genes in K. marxianus and S. cerevisiae. a, Schematic representation of cultivation conditions (1-5, see Fig. 1) in chemostat cultures of $S$. cerevisiae CEN.PK113-7D and $K$. marxianus CBS6556. b, Transcriptional differences in the mevalonate- and ergosterolpathway genes of $S$. cerevisiae and $K$. marxianus. Contrasts indicate the following comparisons: 21: oxygen-limited (840 ppm $\mathrm{O}_{2}$ in inlet gas) and supplementation with Tween 80 and ergosterol versus aerobic, glucose limited; 31 : severely oxygen limited $\left(<0.5 \mathrm{ppm}_{2}\right.$ in inlet gas) versus aerobic and glucose limited; 32 : severely oxygen limited ( $<0.5 \mathrm{ppm} \mathrm{O}$ in inlet gas) versus oxygen limited ( $840 \mathrm{ppm} \mathrm{O}_{2}$ in inlet gas), both supplemented with Tween 80 and ergosterol; 43 (severely oxygen limited ( $<0.5 \mathrm{ppm}_{2}$ in inlet gas), supplemented with only Tween 80 versus severely oxygen limited, supplemented with Tween 80 and ergosterol); 54 : severely oxygen limited ( $<0.5 \mathrm{ppm} \mathrm{O}_{2}$ in inlet gas) without supplementation of Tween 80 or ergosterol versus severely oxygen limited with supplementation of only Tween 80. Lumped biochemical reactions are represented by arrows. Colors indicate up- (blue) or down-regulation (brown) with color intensity indicating log 2 fold differences with color range capped to a maximum of 4 . Reactions are labeled with the corresponding gene name, $K$. marxianus genes are indicated with the name of the S. cerevisiae orthologs. Ergosterol uptake by S. cerevisiae requires additional factors beyond the membrane transporters Aus1 and Pdr11 (Alimardani et al., 2004). No orthologs of the sterol-transporters or Hmg2 were identified for K. marxianus and low read counts for Erg3, Erg9 and Erg20 precluded differential gene expression analysis across all conditions (dark grey). Enzyme abbreviations: Erg10 acetyl-CoA acetyltransferase, Erg13 3-hydroxy-3-methylglutaryl-CoA (HMG-CoA) synthase, Hmg1/Hmg2 HMG-CoA reductase, Erg12 mevalonate kinase, Erg8 phosphomevalonate kinase, Mvd1 mevalonate pyrophosphate decarboxylase, Idi1 isopentenyl diphosphate:dimethylallyl diphosphate (IPP) isomerase, Erg20 farnesyl pyrophosphate synthetase, Erg9 farnesyl-diphosphate transferase (squalene synthase), Erg7 lanosterol synthase, Erg11 lanosterol 14 $\alpha$-demethylase, Cyb5 cytochrome $b 5$ (electron donor for sterol C5-6 desaturation), Ncp1 NADP-cytochrome P450 reductase, Erg24 C-14 sterol reductase, Erg25 C-4 methyl sterol oxidase, Erg26 C-3 sterol dehydrogenase, Erg27 3-keto-sterol reductase, Erg28 endoplasmic reticulum membrane protein (may facilitate protein-protein interactions between Erg26 and Erg27, or tether these to the ER), Erg6 $\Delta 24$-sterol C-methyltransferase, Erg2 $\Delta 24$-sterol C-methyltransferase, Erg3 C-5 sterol desaturase, Erg5 C-22 sterol desaturase, Erg4 C24/28 sterol reductase, Aus1/Pdr11 plasma-membrane sterol transporter. 
a

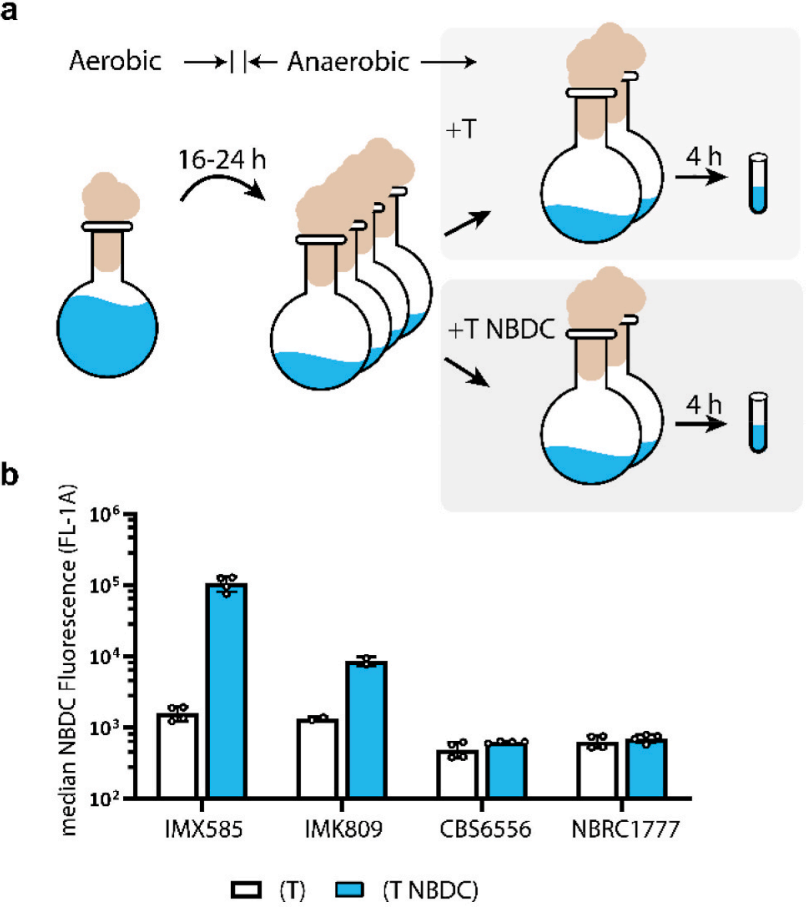

C

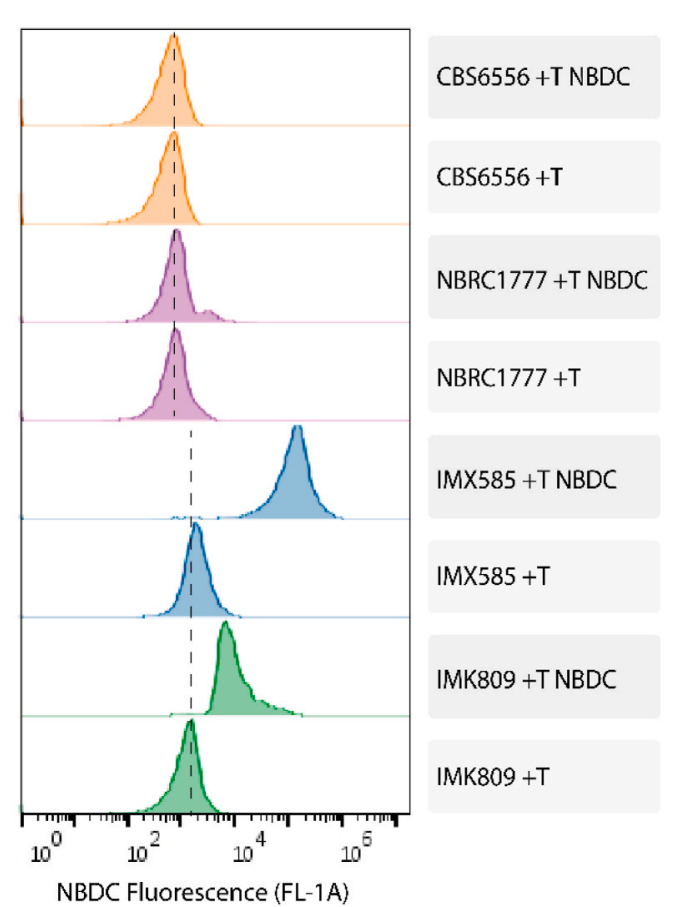

Fig. 4. | Uptake of the fluorescent sterol derivative NBDC by S. cerevisiae and K. marxianus strains. a, Experimental approach. S. cerevisiae strains IMX585 (reference) and IMK809 (aus1 $\Delta$ pdr11 ), and K. marxianus strains NBRC1777 and CBS6556 were each anaerobically incubated in four replicate shake-flask cultures. NBDC and Tween 80 (NBDC T) were added to two cultures, while only Tween 80 (T) was added to the other two. After $4 \mathrm{~h}$ incubation, cells were stained with propidium iodide (PI) and analyzed by flow cytometry. PI staining was used to eliminate cells with compromised membrane integrity from analysis of NBDC fluorescence. Cultivation conditions and flow cytometry gating are described in Methods and in Supplementary Fig. 8, Supplementary Data set 1 and 2. b, Median and pooled standard deviation of fluorescence intensity $\left(\lambda_{\mathrm{ex}} 488 \mathrm{~nm} \mid \lambda_{\mathrm{em}} 533 / 30 \mathrm{~nm}\right.$, FL1-A) of PI-negative cells with variance of biological replicates after $4 \mathrm{~h}$ exposure to Tween 80 (white bars) or Tween 80 and NBDC (blue bars). Variance was pooled for the strains IMX585, CBS6556 and NBRC1777 by repeating the experiment. c, NBDC fluorescence-intensity distribution of cells in a sample from a single culture for each strain, shown as modal-scaled density function. Dashed lines represent background fluorescence of unstained cells of $S$. cerevisiae and K. marxianus. Fluorescence data for 23-h incubations with NBDC are shown in Supplementary Fig. 7.

preculture (Dekker et al., 2019). The tetrahymanol-producing strain did not grow under these conditions (Fig. 5c) but showed sustained growth under severely oxygen-limited conditions that did not support growth of strain NBRC1777 (Fig. 5de). Single-cell isolates derived from these oxygen-limited cultures (IMS1111, IMS1131, IMS1132, IMS1133) showed instantaneous as well as sustained growth under strictly anaerobic conditions (Fig. $5 \mathrm{f}$ and $\mathrm{g}$ ) from an aerobic pre-culture. Tetrahymanol contents in the first, second and third cycle of anaerobic cultivation of isolate IMS1111 were $7.6 \pm 0.0 \mathrm{mg} \mathrm{g}^{-1}, 28.0 \pm 13.0 \mathrm{mg}$ $\mathrm{g}^{-1}$ and $11.5 \pm 0.1 \mathrm{mg} \mathrm{g}^{-1}$, respectively (Fig. 5b), while no ergosterol was detected.

To identify whether adaptation of the tetrahymanol-producing strain IMX2323 to anaerobic growth involved genetic changes, its genome and those of the four adapted isolates were sequenced (Supplementary Table 1). No copy number variations were detected in any of the four adapted isolates. Only strain IMS1111 showed two non-conservative mutations in coding regions: a single-nucleotide insertion in a transposon-borne gene and a stop codon at position 350 (of $496 \mathrm{bp}$ ) in KmCLN3, which encodes for a G1 cyclin (Landry et al., 2012). The apparent absence of mutations in the three other, independently adapted strains indicated that their ability to grow anaerobically reflected a non-genetic adaptation.

\subsection{Selection for faster anaerobic growth and thermotolerance}

One of the attractive phenotypes of $K$. marxianus for industrial application is its high thermotolerance, with reported maximum growth temperatures of $46-52{ }^{\circ} \mathrm{C}$ (Fonseca et al., 2008; Madeira and Gombert, 2018). To test if anaerobically growing tetrahymanol-producing strains retained thermotolerance, strain IMS1111 was grown in anaerobic sequential-batch-reactor (SBR) cultures with a working volume of 100 $\mathrm{mL}$ (Fig. 6). After an initial growth cycle at $30{ }^{\circ} \mathrm{C}$, the growth temperature was shifted to $42{ }^{\circ} \mathrm{C}$. Specific growth at $42{ }^{\circ} \mathrm{C}$ progressively accelerated from $0.06 \mathrm{~h}^{-1}$ to $0.13 \mathrm{~h}^{-1}$ over $17 \mathrm{SBR}$ cycles (corresponding to ca. 65 generations; Fig. $6 \mathrm{~b}$ ). A subsequent temperature increase to $45{ }^{\circ} \mathrm{C}$ led to a decrease of the maximum growth rate which, after approximately 70 cycles of selective growth, stabilized at $0.08 \mathrm{~h}^{-1}$. Whole-population genome sequencing of the evolved populations revealed no common mutations or chromosomal copy number variations (Supplementary Table 1). These data show that TtSTC1-expressing $K$. marxianus can grow anaerobically at temperatures up to at least $45^{\circ} \mathrm{C}$.

\section{Discussion}

Industrial production of ethanol from carbohydrates relies on S. cerevisiae, due to its capacity for efficient, fast alcoholic fermentation and growth under strictly anaerobic process conditions. Many facultatively fermentative yeast species outside the Saccharomycotina WGDclade also rapidly ferment sugars to ethanol under oxygen-limited conditions (Dashko et al., 2014), but cannot grow and ferment in the complete absence of oxygen (Merico et al., 2007; Snoek and Steensma, 2006; Visser et al., 1990). Identifying and eliminating oxygen requirements of these yeasts is essential to unlock their industrially relevant traits for application. Here, this challenge was addressed for the thermotolerant yeast $K$. marxianus, using a systematic approach based on chemostat-based quantitative physiology, genome and transcriptome analysis, sterol-uptake assays and genetic modification. S. cerevisiae, which was used as a reference in this study, shows strongly different genome-wide expression profiles under aerobic and anaerobic or 
a

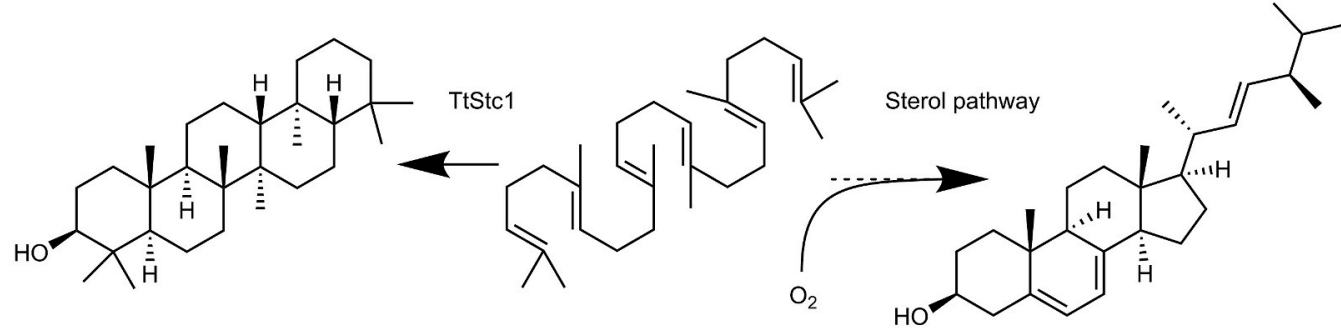

b

Tetrahymanol

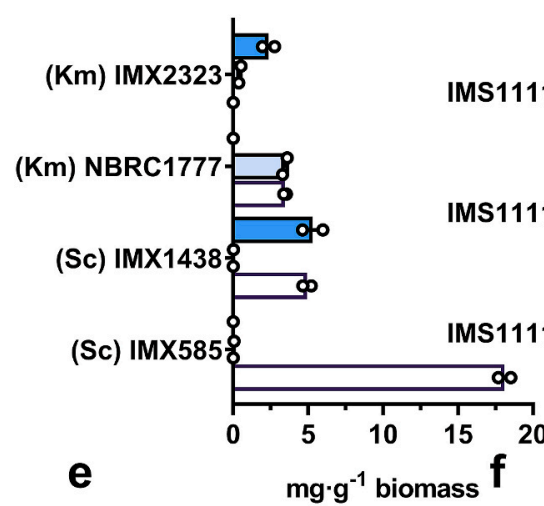

Squalene
C

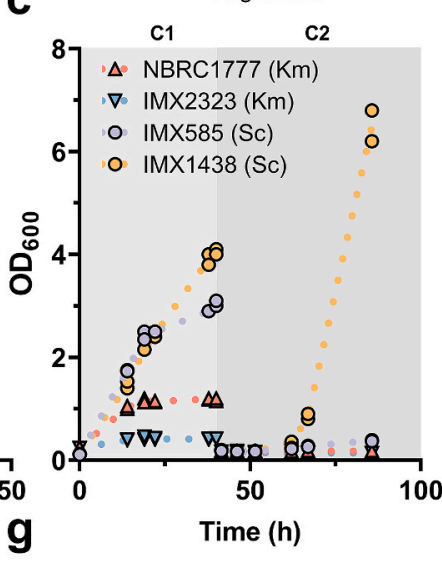

d

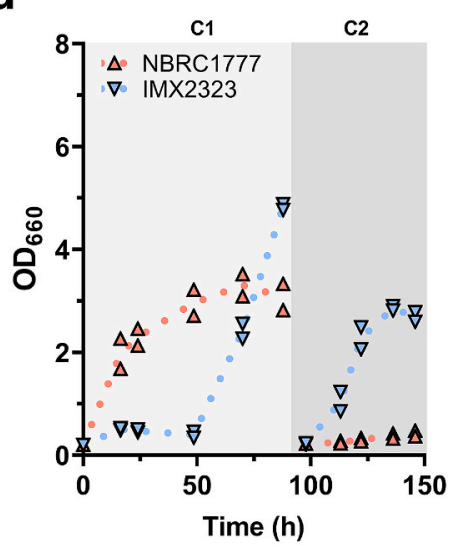

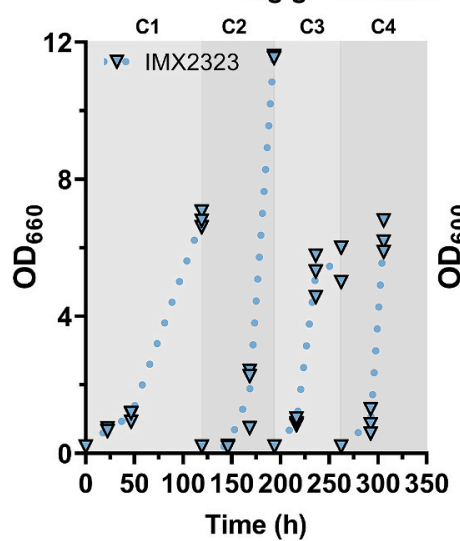
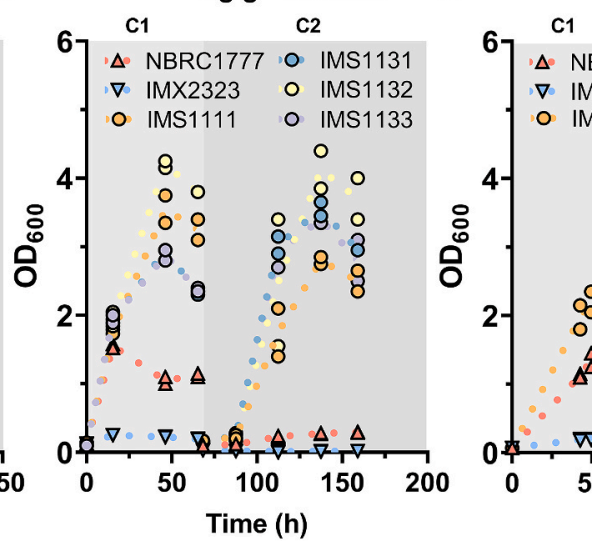

C2

C3

C4

C5

(1777

IMX2323

IMS1111

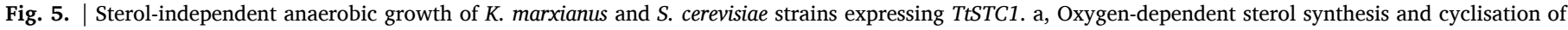

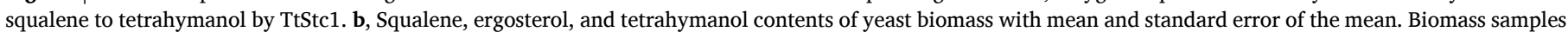

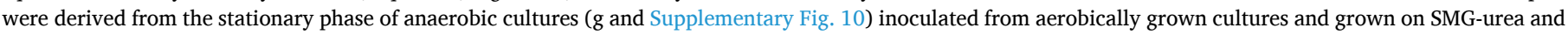

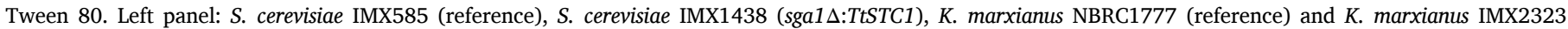

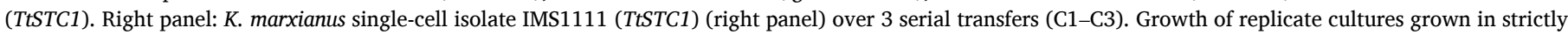

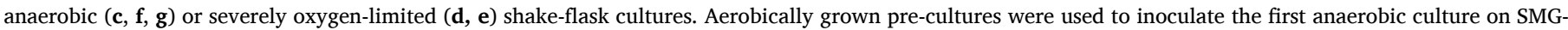

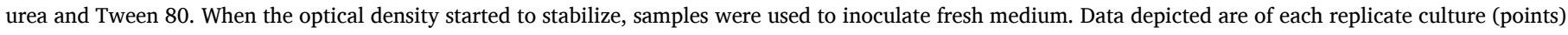

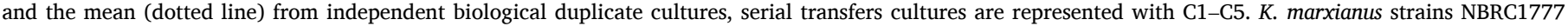

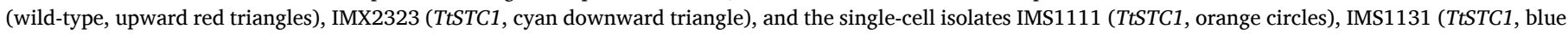

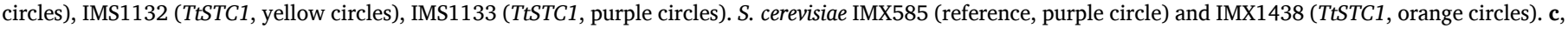
Extended data with double inoculum size is available in Supplementary Fig. 10 d, Extended data are available in Supplementary Fig. 9a.

oxygen-limited conditions (Tai et al., 2005). Although only a small fraction of these differences were conserved in K. marxianus (Fig. 2), we were able to identify absence of a functional sterol import system as the critical cause for its inability to grow anaerobically. Enabling synthesis of the sterol surrogate tetrahymanol yielded strains that grew anaerobically at temperatures above the permissive temperature range of S. cerevisiae.

A short adaptation phase of tetrahymanol-producing $K$. marxianus strains under oxygen-limited conditions reproducibly enabled strictly anaerobic growth, which was retained after aerobic isolation of singlecell lines. However, resequencing of the genomes of thus adapted strains did not identify potential causal mutations. In this context, it may be relevant to note that, in contrast to wild-type $K$. marxianus, a nonadapted tetrahymanol-producing strain did not show 'carry-over growth' after transfer from aerobic to strictly anaerobic conditions. In addition, anaerobic cultures of adapted TtSTC1-expressing K. marxianus strains showed reduced squalene contents (Fig. 5). A similar reduction of squalene content was observed during anaerobic SBR cultivation of an TtSTC1-expressing S. cerevisiae strain (Wiersma et al., 2020). Based on these observations, we speculate that relative contents of tetrahymanol and squalene may be critical for the onset of anaerobic growth. Once microaerobic adaptation has established a balance between these lipids that is permissive for anaerobic growth, this might then be stabilized by native regulation networks. Genome-wide expression studies on adapted 
a

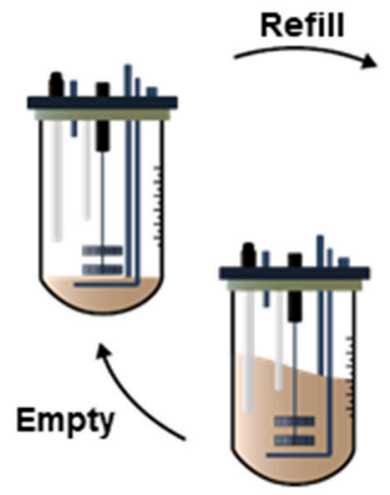

b

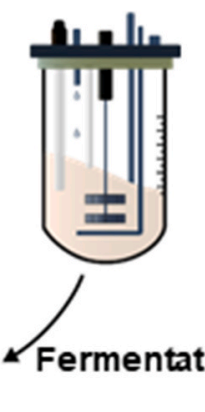

(1)

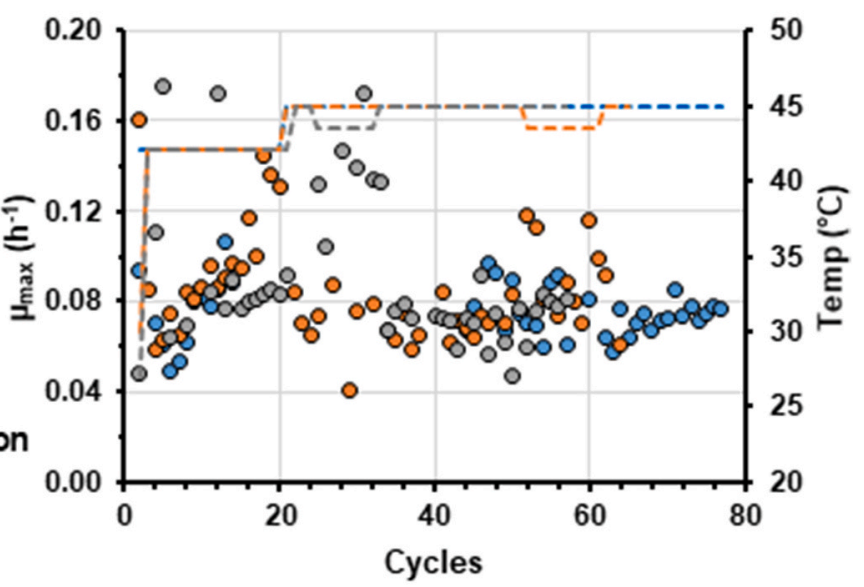

C

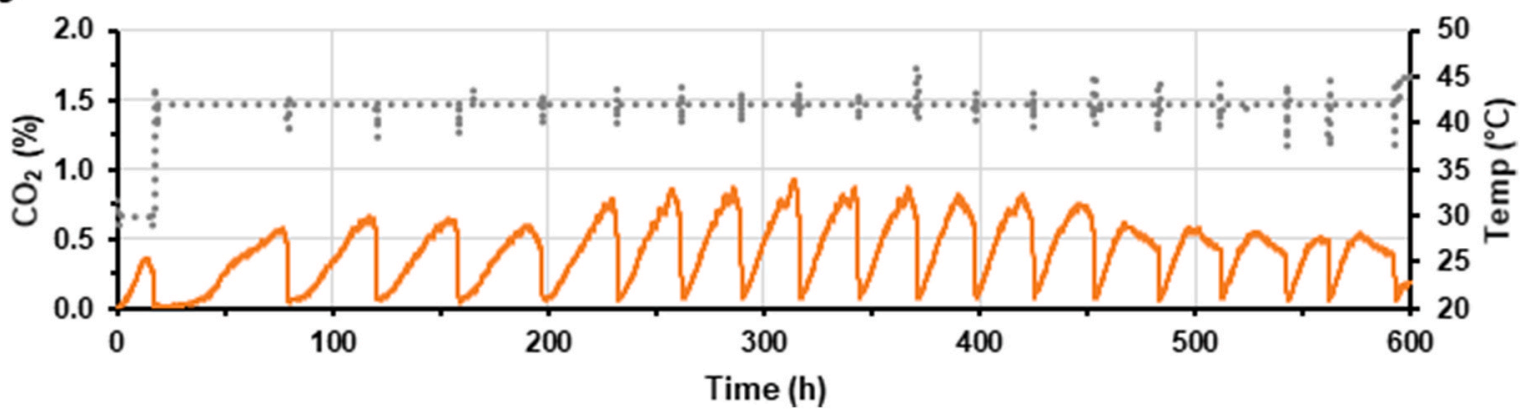

Fig. 6. Thermotolerance and anaerobic growth of tetrahymanol-producing $K$. marxianus strain. The strain IMS1111 was grown in triplicate sequential batch reactor (SBR) cultures with a working volume of $100 \mathrm{~mL}$ in synthetic media supplemented with $20 \mathrm{~g} \mathrm{~L}^{-1}$ glucose and $420 \mathrm{mg} \mathrm{L}^{-1}$ Tween 80 at pH 5.0. a, Experimental design of sequential batch fermentation with cycles at stepwise increasing temperatures to select for faster growing mutants, each cycle consisted of three phases; (i) (re) filling of the bioreactor with fresh media up to $100 \mathrm{~mL}$ and adjustment of temperature to a new set-point, (ii) anaerobic batch fermentation at a fixed culture temperature with continuous $\mathrm{N}_{2}$ sparging for monitoring of $\mathrm{CO}_{2}$ in the culture off-gas, and (iii) fast broth withdrawal leaving $7 \mathrm{~mL}$ ( $14.3 \mathrm{fold}$ dilution $\approx 3.8$ generations) to inoculate the next batch. b. Maximum specific estimated growth rate (circles) of each batch cycle for the three independent bioreactor cultivations (M3R blue, M5R orange, M6L grey). The growth rate was calculated from the $\mathrm{CO}_{2}$ production as measured in the off-gas and should be interpreted as an estimate and in some cases could not be calculated. The culture temperature profile (dotted line) for each independent bioreactor cultivation (blue, grey, orange) consisted of a stepwise increment of the temperature at the onset of the fermentation phase in each batch cycle. c, Representative section of $\mathrm{CO}_{2}$ off-gas profiles of the individual bioreactor (M5R) cultivation over time with $\mathrm{CO}_{2}$ fraction (orange line) and culture temperature (grey dotted line), data of the entire experiment is available in Supplementary Fig. 11.

and non-adapted cultures and modification of intracellular squalene pools, may elucidate how tetrahymanol interacts with other membrane components and whether such interactions are involved in adaptation to anaerobic growth of tetrahymanol-producing $K$. marxianus.

Comparative genomic studies in Saccharomycotina yeasts have previously led to the hypothesis that sterol transporters are absent from pre-WGD yeast species (Seret et al., 2009; Snoek and Steensma, 2006). While our observations on $K$. marxianus reinforce this hypothesis, which was hitherto not experimentally tested, they do not exclude involvement of additional oxygen-requiring reactions in other non-Saccharomyces yeasts. For example, pyrimidine biosynthesis is often cited as a key oxygen-requiring process in non-Saccharomyces yeasts, due to involvement of a respiratory-chain-linked dihydroorotate dehydrogenase (DHOD) (Gojković et al., 2004; Shi and Jeffries, 1998). K. marxianus, is among a small number of yeast species that, in addition to this respiration dependent enzyme (KmUra9), also harbors a fumarate-dependent DHOD (KmUra1) (Riley et al., 2016). In K. marxianus the activation of this oxygen-independent KmUra1 is a crucial adaptation for anaerobic pyrimidine biosynthesis. The experimental approach followed in the present study should be applicable to resolve the role of pyrimidine biosynthesis and other oxygen-requiring reactions in additional yeast species.

Enabling K. marxianus to grow anaerobically represents an important step towards application of this thermotolerant yeast in large-scale anaerobic bioprocesses. However, specific growth rates of anaerobic SBR cultures of TtSTC1-expressing $K$. marxianus strains growing at $45^{\circ} \mathrm{C}$
Table 2

Strains used in this study. Abbreviations: Saccharomyces cerevisiae (Sc), Kluyveromyces marxianus $(\mathrm{Km})$, Tetrahymena thermophila $(\mathrm{Tt})$.

\begin{tabular}{|c|c|c|c|}
\hline Genus & Strain & Relevant genotype & Reference \\
\hline S. cerevisiae & $\begin{array}{l}\text { CEN.PK113- } \\
\text { 7D }\end{array}$ & $\begin{array}{l}\text { MATa URA3 HIS3 LEU2 } \\
\text { TRP1 MAL2-8c SUC2 }\end{array}$ & $\begin{array}{l}\text { Entian and Kötter } \\
\text { (2007) }\end{array}$ \\
\hline S. cerevisiae & IMX585 & $\begin{array}{l}\text { CEN.PK113-7D can1 } 1 \Delta: \text { :cas9- } \\
\text { natNT2 }\end{array}$ & Mans et al. (2015) \\
\hline S. cerevisiae & IMX1438 & IMX585 sga1 1 :::TtSTC1 & $\begin{array}{l}\text { Wiersma et al. } \\
(2020)\end{array}$ \\
\hline S. cerevisiae & IMK802 & IMX585 aus1 $1 \Delta$ & This study \\
\hline S. cerevisiae & IMK806 & IMX585 pdr11 $\Delta$ & This study \\
\hline S. cerevisiae & IMK809 & IMX585 aus1 $1 \Delta$ pdr11 & This study \\
\hline K. marxianus & CBS6556 & URA3 HIS3 LEU2 TRP1 & CBS-KNAW \\
\hline K. marxianus & NBRC1777 & URA3 HIS3 LEU2 TRP1 & NBRC \\
\hline K. marxianus & IMX2323 & $\begin{array}{l}\text { KmPDC1p-TtSTC1-ScADH1t- } \\
\text { hygB }\end{array}$ & This study \\
\hline K. marxianus & IMS1111 & $\begin{array}{l}\text { KmPDC1p-TtSTC1-ScADH1t- } \\
\text { hygB }\end{array}$ & This study \\
\hline K. marxianus & IMS1112 & $\begin{array}{l}\text { KmPDC1p-TtSTC1-ScADH1t- } \\
\text { hygB }\end{array}$ & This study \\
\hline K. marxianus & IMS1113 & $\begin{array}{l}\text { KmPDC1p-TtSTC1-ScADH1t- } \\
\text { hygB }\end{array}$ & This study \\
\hline K. marxianus & IMS1131 & $\begin{array}{l}\text { KmPDC1p-TtSTC1-ScADH1t- } \\
\text { hygB }\end{array}$ & This study \\
\hline K. marxianus & IMS1132 & $\begin{array}{l}\text { KmPDC1p-TtSTC1-ScADH1t- } \\
\text { hygB }\end{array}$ & This study \\
\hline K. marxianus & IMS1133 & $\begin{array}{l}\text { KmPDC1p-TtSTC1-ScADH1t- } \\
\text { hygB }\end{array}$ & This study \\
\hline
\end{tabular}


Table 3

Plasmids used in this study. Restriction enzyme recognition sites are indicated in superscript. US/DS represent upstream and downstream homologous recombination sequences used for genomic integration into the $K$. marxianus lac 4 locus. Abbreviations: Saccharomyces cerevisiae (Sc), Kluyveromyces marxianus (Km), Tetrahymena thermophila (Tt).

\begin{tabular}{|c|c|c|}
\hline Plasmid & Characteristics & Source \\
\hline pGGkd015 & ori ampR ConLS GFP ConR1 & $\begin{array}{l}\text { Hassing et al. } \\
\text { (2019) }\end{array}$ \\
\hline pGGKd068 & $\begin{array}{l}\text { ori kanR }{ }^{\text {NotI }} \text { Kmlac4 }_{\mathrm{Us}}{ }^{\text {BsmBI }}{ }^{\text {ConRE }}{ }^{\text {BsaI }} \text { sfGFP }^{\text {BsaI }} \\
\text { ConLS }{ }^{\text {BsmBI }} \text { hygB Kmlac4 } 4_{\text {DS }}^{\text {Not }}\end{array}$ & This study \\
\hline pP2 & ori cam ${ }^{\mathrm{R}} K m P D C 1 \mathrm{p}$ & $\begin{array}{l}\text { Rajkumar et al. } \\
\text { (2019) }\end{array}$ \\
\hline pROS11 & $\begin{array}{l}\text { ori amp }^{\mathrm{R}} 2 \mu \mathrm{m} \text { amdSYM pSNR52-gRNA } \\
\text { pRSNR } \text { 2-gRNA } \\
A D E 2\end{array}$ & $\begin{array}{l}\text { Mans et al. } \\
(2015)\end{array}$ \\
\hline pUD696 & ori kanR TtSTC1 & $\begin{array}{l}\text { Wiersma et al. } \\
\text { (2020) }\end{array}$ \\
\hline pUDE659 & $\begin{array}{l}\text { ori amp }{ }^{\mathrm{R}} 2 \mu \mathrm{m} \text { amdSYM pSNR52-gRNA } \\
\text { pRSNRS1 } \text {-gRNA } \\
A U S 1\end{array}$ & This study \\
\hline pUDE663 & $\begin{array}{l}\text { ori amp }^{\mathrm{R}} 2 \mu \mathrm{m} \text { amdSYM pSNR52-gRNA } \\
\text { pRSNRE11 } \text {-gRNA } \\
P D R 11\end{array}$ & This study \\
\hline pUDE909 & ori ampR KmPDC1p-TtSTC1-ScADH1t & This study \\
\hline pUDI246 & $\begin{array}{l}\text { ori kanR }{ }^{\text {NotI }}{ }_{\text {Kmlac4 }} \text { Us KmPDC1p-TtSTC1-ScADH1t } \\
\text { hygB Kmlac4 } 4_{\mathrm{DS}}^{\text {NoI }}\end{array}$ & This study \\
\hline pYTK008 & ori camR ConLS & Lee et al. (2015) \\
\hline pYTK047 & ori camR GFP dropout & Lee et al. (2015) \\
\hline pYTK053 & ori camR $S c A D H 1 \mathrm{t}$ & Lee et al. (2015) \\
\hline pYTK073 & ori camR ConRE' & Lee et al. (2015) \\
\hline pYTK079 & ori camR hygB & Lee et al. (2015) \\
\hline
\end{tabular}

were only approximately $0.08 \mathrm{~h}^{-1}$, which is ca. 4-fold lower than the anaerobic growth rate of $S$. cerevisiae in anaerobic, glucose-grown batch cultures grown at $30{ }^{\circ} \mathrm{C}$ (Dekker et al., 2019; Papapetridis et al., 2018). In addition, final optical densities of anaerobic batch cultures of TtStc1-expressing $K$. marxianus cultures at $30{ }^{\circ} \mathrm{C}\left(\mathrm{OD}_{600}\right.$ of approximately 3, Fig. $5 \mathrm{~g}$ ) were over two-fold lower than those of Tween 80 and ergosterol-supplemented $S$. cerevisiae cultures grown

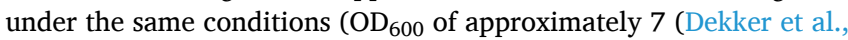
2019),). A similar phenotype of tetrahymanol-producing S. cerevisiae was proposed to reflect an increased membrane permeability (Wiersma et al., 2020). Additional membrane engineering or expression of a functional sterol transport system is therefore required for further development of robust, anaerobically growing industrial strains of $K$. marxianus (Guo et al., 2020).

\section{Methods}

\subsection{Yeast strains, maintenance and shake-flask cultivation}

Yeast strains used and constructed in this study (Table 2). Saccharomyces cerevisiae CEN.PK113-7D (Entian and Kötter, 2007; Nijkamp et al., 2012) (MATa MAL2-8c SUC2) was obtained from Dr. Peter Kötter, J.W. Goethe University, Frankfurt. Kluyveromyces marxianus strains CBS 6556 (ATCC 26548; NCYC 2597; NRRL Y-7571) and NBRC 1777 (IFO 1777) were obtained from the Westerdijk Fungal Biodiversity Institute (CBS-KNAW, Utrecht, The Netherlands) and the

Table 4

CRISPR gRNA target sequences used in this study. gRNA target sequences are shown with PAM sequences underlined. Position in ORF indicates the base pair after which the Cas9-mediated double-strand break is introduced. AT score indicates the AT content of the 20-bp target sequence and RNA score indicates the fraction of unpaired nucleotides of the 20-bp target sequence, predicted with the complete gRNA sequence using a minimum free energy prediction by the RNAfold algorithm (Lorenz et al., 2011).

\begin{tabular}{lllll}
\hline Locus & Target sequence $\left(5^{\prime}-3^{\prime}\right)$ & $\begin{array}{l}\text { Position in } \\
\text { ORF }(\mathrm{bp})\end{array}$ & $\begin{array}{l}\text { AT } \\
\text { score }\end{array}$ & $\begin{array}{l}\text { RNA } \\
\text { score }\end{array}$ \\
\hline AUS1 & CATTATTGTAAATGATTTGGTGG & $320 / 4184$ & 0.75 & 1 \\
PDR11 & ATCTTTCATATAAATAACATAGG & $1627 / 4235$ & 0.85 & 1 \\
\hline
\end{tabular}

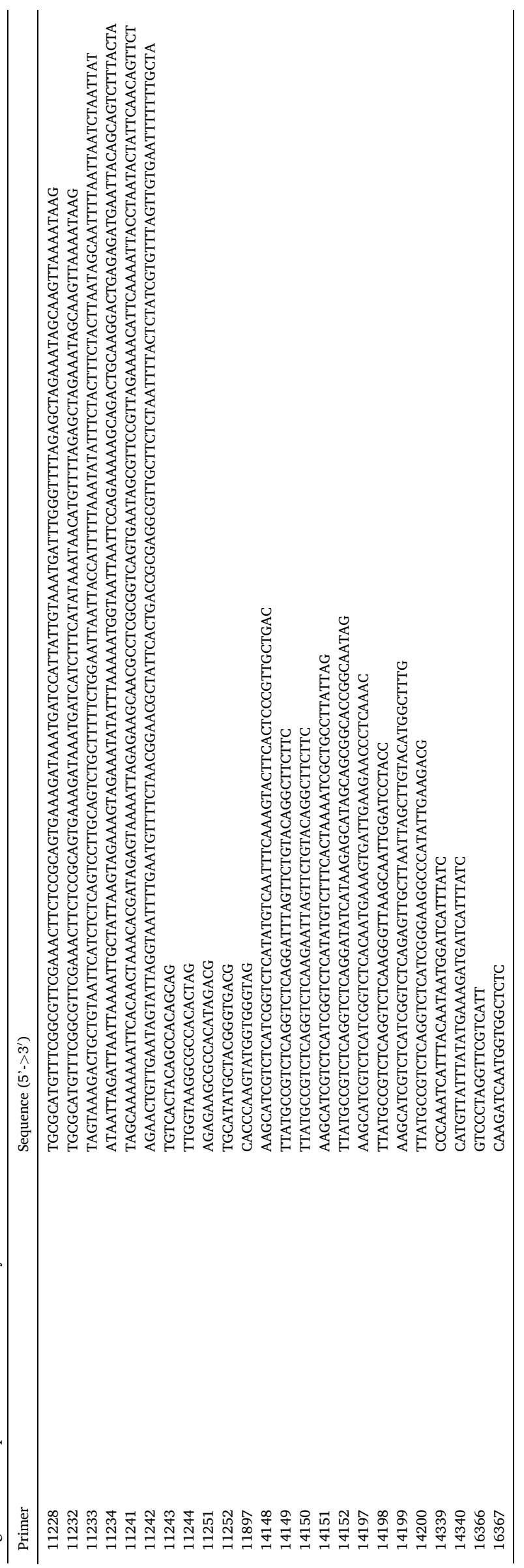


Biological Resource Center, NITE (NBRC) (Chiba, Japan), respectively. Stock cultures of $S$. cerevisiae were grown at $30{ }^{\circ} \mathrm{C}$ in an orbital shaker set at $200 \mathrm{rpm}$, in $500 \mathrm{~mL}$ shake flasks containing $100 \mathrm{~mL}$ YPD $\left(10 \mathrm{~g} \mathrm{~L}^{-1}\right.$ Bacto yeast extract, $20 \mathrm{~g} \mathrm{~L}^{-1}$ Bacto peptone, $20 \mathrm{~g} \mathrm{~L}^{-1}$ glucose). For cultures of $K$. marxianus, the glucose concentration was reduced to $7.5 \mathrm{~g}$ $\mathrm{L}^{-1}$. After addition of glycerol to early stationary-phase cultures, to a concentration of $30 \%(\mathrm{v} / \mathrm{v}), 2 \mathrm{~mL}$ aliquots were stored at $-80{ }^{\circ} \mathrm{C}$. Shake-flask precultures for bioreactor experiments were grown in 100 $\mathrm{mL}$ synthetic medium (SM) with glucose as carbon source and urea as nitrogen source (SMG-urea) (Bracher et al., 2017; Verduyn et al., 1990). For anaerobic cultivation, synthetic medium was supplemented with ergosterol $\left(10 \mathrm{mg} \mathrm{L}^{-1}\right)$ and Tween $80\left(420 \mathrm{mg} \mathrm{L}^{-1}\right)$ as described previously (Andreasen and Stier, 1953a; Dekker et al., 2019; Verduyn et al., 1990).

\subsection{Expression-cassette and plasmid construction}

Plasmids used in this study are described in (Table 3). To delete the sterol transporters AUS1 and PDR1 in S. cerevisiae with CRISPR-Cas9, the gRNA-expression plasmids pUDE659 ( (RRNA $_{A U S 1}$ ) and pUDE663 $\left(\right.$ RRNA $\left._{P D R 11}\right)$ were constructed with target sequence described in (Table 4). To this end, the pROS11 plasmid-backbone was PCR amplified using Phusion HF polymerase (Thermo Scientific, Waltham, MA) with the double-binding primer 6005. PCR amplifications were performed with desalted or PAGE-purified oligonucleotide primers (Sigma-Aldrich, St Louis, MO) according to manufacturer's instructions (see Table 5). To introduce the gRNA-encoding nucleotide sequences into gRNAexpression plasmids, a $2 \mu \mathrm{m}$ fragment was first amplified with primers 11228 and 11232 containing the specific sequence as primer overhang using pROS11 as template. PCR products were purified with genElute PCR Clean-Up Kit (Sigma-Aldrich) or Gel DNA Recovery Kit (Zymo Research, Irvine, CA). The two DNA fragments were then assembled by Gibson Assembly (New England Biolabs, Ipswich, MA) according to the manufacturer's instructions. Gibson assembly reaction volumes were downscaled to $10 \mu \mathrm{L}$ and $0.01 \mathrm{pmol} \mu \mathrm{L}^{-1}$ DNA fragments at $1: 1 \mathrm{M}$ ratio for $1 \mathrm{~h}$ at $50^{\circ} \mathrm{C}$. Chemically competent $E$. coli XL1-Blue was transformed with the Gibson assembly mix via a 5 min incubation on ice followed by a $40 \mathrm{~s}$ heat shock at $42{ }^{\circ} \mathrm{C}$ and $1 \mathrm{~h}$ recovery in non-selective LB medium. Transformants were selected on LB agar containing the appropriate antibiotic. Golden Gate assembly with the yeast tool kit (Lee et al., 2015) was performed in $20 \mu \mathrm{L}$ reaction mixtures containing $0.75 \mu \mathrm{L}$ BsaI HF V2 (NEB, \#R3733), $2 \mu \mathrm{L}$ DNA ligase buffer with ATP (New England Biolabs), $0.5 \mu \mathrm{L}$ T7-ligase (NEB) with $20 \mathrm{fmol}$ DNA donor fragments and MilliQ water. Before ligation at $16{ }^{\circ} \mathrm{C}$ was initiated by addition of T7 DNA ligase, an initial BsaI digestion ( $30 \mathrm{~min}$ at $37^{\circ} \mathrm{C}$ ) was performed. Then 30 cycles of digestion and ligation at $37^{\circ} \mathrm{C}$ and $16^{\circ} \mathrm{C}$, respectively, were performed, with $5 \mathrm{~min}$ incubation times for each reaction. Thermocycling was terminated with a $5 \mathrm{~min}$ final digestion step at $60{ }^{\circ} \mathrm{C}$.

To construct a TtSTC1 expression vector for integration into the K. marxianus genome, the coding sequence of TtSTC1 (pUD696) was PCR amplified with primer pair 16096/16097 and Golden gate assembled with the donor plasmids pGGkd015 (ori ampR), pP2 (KmPDC1p), pYTK053 (ScADH1t) resulting in pUDE909 (ori ampR KmPDC1pTtSTC1-ScADH1t). For integration of TtSTC1 cassette into the $K$. marxianus genome upstream and downstream flanking sequences (877/878 bps) of the lac4 locus were PCR amplified with the primer pairs 14197/14198 and 14199/14200, respectively. An empty integration vector, pGGKd068, was constructed by BsaI golden gate cloning of pYTK047 (GFP-dropout), pYTK079 (hygB), pYTK090 (kanR), pYTK073 (ConRE'), pYTK008 (ConLS') together with the two lac4 homologous nucleotide sequences. Plasmid assembly was verified by PCR amplification with primers 15210, 9335, 16274 and 16275 and by digestion with BsmBI (New England Biolabs, \#R0580). The integration vector pUDI246 with the TtSTC1 expression cassette was constructed by Gibson assembly of the PCR amplified pGGKd068 and pUDE909 with primer pairs $16274 / 16275$ and $16272 / 16273$, thereby adding 20 bp overlaps for assembly. For this step, the incubation time of the Gibson assembly was increased to $90 \mathrm{~min}$. Plasmid assembly was verified by diagnostic PCR amplification using DreamTaq polymerase (Thermo Scientific) with primers 5941, 8442, 15216 and subsequent Illumina short-read sequencing.

\subsection{Strain construction}

The lithium-acetate/polyethylene-glycol method was used for yeast transformation (Gietz and Woods, 2001). Homologous repair (HR) DNA fragments for markerless CRISPR-Cas9-mediated gene deletions in S. cerevisiae were constructed by annealing two 120 bp primers, using primer pairs 11241/11242 and 11233/11234 for deletion of PDR11 and AUS1, respectively. After transformation of $S$. cerevisiae IMX585 with gRNA plasmids pUDE659 and pUDE663 and double-stranded repair fragments, transformants were selected on synthetic medium with acetamide as sole nitrogen source (Solis-Escalante et al., 2013). Deletion of AUS1 and PDR11 was confirmed by PCR amplification with primer pairs 11243/11244 and 11251/11252, respectively. Loss of gRNA plasmids was induced by cultivation of single-colony isolates on YPD, after which plasmid loss was assessed by absence of growth of single-cell isolates on synthetic medium with acetamide as nitrogen source. An aus1s pdr11s double-deletion strain was similarly constructed by chemical transformation of $S$. cerevisiae IMK802 with pUDE663 and repair DNA. To integrate a TtSTC1 expression cassette into the K. marxianus lac4 locus, K. marxianus NBRC1777 was transformed using the same lithium-acetate/polyethylene-glycol method with $2 \mu \mathrm{g}$ DNA NotI-digested pUDI246. After centrifugation, cells were resuspended in YPD and incubated at $30^{\circ} \mathrm{C}$ for $3 \mathrm{~h}$. Cells were then again centrifuged, resuspended in demineralized water and plated on $200 \mu \mathrm{g} \mathrm{L^{-1 }}$ hygromycin B (InvivoGen, Toulouse, France) containing agar with 40 $\mu \mathrm{g} \mathrm{L}^{-1}$ X-gal, 5-bromo-4-chloro-3-indolyl- $\beta$-D-galactopyranoside (Fermentas, Waltham, MA). Diagnostic PCR of colonies with primers 16366, 16367 and 11897 indicated that, rather than at the lac4 locus, genomic integration of TtSTC1 had occurred elsewhere in the genome. Chromosomal integration outside lac4 was confirmed for one of the transformants, K. marxianus IMX2323 (TtSTC1), by short-read Illumina sequencing (see Data availability).

\subsection{Chemostat cultivation}

Chemostat cultures were grown at $30^{\circ} \mathrm{C}$ in $2 \mathrm{~L}$ bioreactors (Applikon, Delft, the Netherlands) with a stirrer speed of $800 \mathrm{rpm}$. The dilution rate was set at $0.10 \mathrm{~h}^{-1}$ and a constant working volume of $1.2 \mathrm{~L}$ was maintained by connecting the effluent pump to a level sensor. Cultures were grown on synthetic medium with vitamins (Verduyn et al., 1990). Concentrated glucose solutions were autoclaved separately at $110^{\circ} \mathrm{C}$ for $20 \mathrm{~min}$ and added to the sterile media along with sterile Pluronic 6100 $\mathrm{PE}$ antifoam (BASF, Ludwigshafen, Germany; final concentration $0.2 \mathrm{~g}$ $\mathrm{L}^{-1}$ ). To compensate for a lower biomass yield of fermenting yeast cultures on glucose, media for oxygen-limited chemostat cultivation were supplemented with a higher glucose concentration $\left(25 \mathrm{~g} \mathrm{~L}^{-1}\right)$ than fully aerobic cultures $\left(7.5 \mathrm{~g} \mathrm{~L}^{-1}\right)$. Before autoclaving, bioreactors were tested for gas leakage by submerging them in water while applying a 0.3 bar overpressure.

Anaerobic conditions of bioreactor cultivations were maintained by continuous reactor headspace aeration with pure nitrogen gas $(\leq 0.5$ ppm $\mathrm{O}_{2}$, HiQ Nitrogen 6.0, Linde AG, Schiedam, the Netherlands) at a flowrate of $500 \mathrm{~mL} \mathrm{~N}_{2} \mathrm{~min}^{-1}(2.4 \mathrm{vvm})$. Gas pressure of $1.2 \mathrm{bar}$ of the reactor headspace was set with a reduction valve (Tescom Europe, Hannover, Germany) and remained constant during cultivation. To prevent oxygen diffusion into the cultivation the bioreactor was equipped with Fluran tubing (14 Barrer $\mathrm{O}_{2}$, F-5500-A, Saint-Gobain, Courbevoie, France), Viton O-rings (Eriks, Alkmaar, the Netherlands), and no $\mathrm{pH}$ probes were mounted. The medium reservoir was deoxygenated by sparge aeration with nitrogen gas $\left(\leq 1 \mathrm{ppm} \mathrm{O}_{2}\right.$, HiQ Nitrogen 5.0, Linde 
AG).

For aerobic cultivation the reactor was sparged continuously with dried air at a flowrate of $500 \mathrm{~mL}$ air $\mathrm{min}^{-1}(2.4 \mathrm{vvm})$. Dissolved oxygen levels were analyzed by Clark electrodes (AppliSens, Applikon) and remained above $40 \%$ during the cultivation. For micro-aerobic cultivation, nitrogen ( $\leq 1 \mathrm{ppm} \mathrm{O}_{2}$, HiQ Nitrogen 5.0, Linde AG) and air were mixed continuously by controlling the fractions of mass flow rate of the dry gas to a total flow of $500 \mathrm{~mL} \mathrm{~min}{ }^{-1}$ per bioreactor. The mixed gas was distributed to each bioreactor and analyzed separately in real-time. Continuous cultures were assumed to be in steady state when after at least 5 vol changes, culture dry weight and the specific carbon dioxide production rates changed by less than $10 \%$.

Cell density was routinely measured at a wavelength of $660 \mathrm{~nm}$ with spectrophotometer Jenway 7200 (Cole Palmer, Staffordshire, UK). Cell dry weight of the cultures were determined by filtering exactly $10 \mathrm{~mL}$ of culture broth over pre-dried and weighed membrane filters $(0.45 \mu \mathrm{m}$, Thermo Fisher Scientific), which were subsequently washed with demineralized water, dried in a microwave oven $(20 \mathrm{~min}, 350 \mathrm{~W})$ and weighed again (Postma et al., 1989).

\subsection{Metabolite analysis}

For determination of substrate and extracellular metabolite concentrations, culture supernatants were obtained by centrifugation of culture samples ( $5 \mathrm{~min}$ at $13000 \mathrm{rpm}$ ) and analyzed by highperformance liquid chromatography (HPLC) on a Waters Alliance 2690 HPLC (Waters, MA, USA) equipped with a Bio-Rad HPX-87H ion exchange column (BioRad, Veenendaal, the Netherlands) operated at $60{ }^{\circ} \mathrm{C}$ with a mobile phase of $5 \mathrm{mM} \mathrm{H}_{2} \mathrm{SO}_{4}$ at a flow rate of $0.6 \mathrm{~mL} \mathrm{~min}^{-1}$. Compounds were detected by means of a dual-wavelength absorbance detector (Waters 2487) and a refractive index detector (Waters 2410) and compared to reference compounds (Sigma-Aldrich). Residual glucose concentrations in continuous cultivations were determined by HPLC analysis from rapid quenched culture samples with cold steel beads (Mashego et al., 2003).

\subsection{Gas analysis}

The off-gas from bioreactor cultures was cooled with a condenser $\left(2{ }^{\circ} \mathrm{C}\right)$ and dried with PermaPure Dryer (Inacom Instruments, Veenendaal, the Netherlands) prior to analysis of the carbon dioxide and oxygen fraction with a Rosemount NGA 2000 Analyser (Baar, Switzerland). The Rosemount gas analyzer was calibrated with defined mixtures of $1.98 \% \mathrm{O}_{2}, 3.01 \% \mathrm{CO}_{2}$ and high-quality nitrogen gas $\mathrm{N} 6$ (Linde AG).

\subsection{Ethanol evaporation rate}

To correct for ethanol evaporation in the continuous bioreactor cultivations the ethanol evaporation rate was determined in the same experimental bioreactor set-up without the yeast. To SM glucose media with urea $400 \mathrm{mM}$ of ethanol was added after which the decrease in the ethanol concentration was measured over time by periodic measurements and quantification by HPLC analysis over the course of at least $140 \mathrm{~h}$. To reflect the media composition used for the different oxygen regimes and anaerobic growth factor supplementation, the ethanol evaporation was measured for bioreactor sparge aeration with Tween 80 , bioreactor head-space aeration both with and without Tween 80 . The ethanol evaporation rate was measured for each condition in triplicate.

\subsection{Lipid extractions \& GC analysis}

For analysis of triterpene and triterpenoid cell contents biomass was harvested, washed once with demineralized water and stored as pellet at $-80{ }^{\circ} \mathrm{C}$ before freeze-drying the pellets using an Alpha 1-4 LD Plus
(Martin Christ, Osterode am Harz, Germany) at $-60^{\circ} \mathrm{C}$ and 0.05 mbar. Freeze-dried biomass was saponificated with $2.0 \mathrm{M} \mathrm{NaOH}$ (Bio-Ultra, Sigma-Aldrich) in methylation glass tubes (PYREX ${ }^{\mathrm{TM}}$ Boroslicate glass, Thermo Fisher Scientific) at $70{ }^{\circ} \mathrm{C}$. As internal standard $5 \alpha$-cholestane (Sigma-Aldrich) was added to the saponified biomass suspension. Subsequently tert-butyl-methyl-ether (tBME, Sigma-Aldrich) was added for organic phase extraction. Samples were extracted twice using tBME and dried with sodium-sulfate (Merck, Darmstadt, Germany) to remove remaining traces of water. The organic phase was either concentrated by evaporation with $\mathrm{N}_{2}$ gas aeration or transferred directly to an injection vial (VWR International, Amsterdam, the Netherlands). The contents were measured by GC-FID using Agilent 7890A Gas Chromatograph (Agilent Technologies, Santa Clara, CA) equipped with an Agilent CP9013 column (Agilent). The oven was programmed to start at $80{ }^{\circ} \mathrm{C}$ for $1 \mathrm{~min}$, ramp first to $280^{\circ} \mathrm{C}$ with $60^{\circ} \mathrm{C} \cdot \mathrm{min}^{-1}$ and secondly to $320^{\circ} \mathrm{C}$ with a rate of $10^{\circ} \mathrm{C} \cdot \mathrm{min}^{-1}$ with a final temperature hold of $15 \mathrm{~min}$. Spectra were compared to separate calibration lines of squalene, ergosterol, $\alpha$-cholestane, cholesterol and tetrahymanol as described previously (Wiersma et al., 2020).

\subsection{Sterol uptake assay}

Sterol uptake was monitored by the uptake of fluorescently labeled 25-NBD-cholesterol (Avanti Polar Lipids, Alabaster, AL). A stock solution of 25-NBD-cholesterol (NBDC) was prepared in ethanol under an argon atmosphere and stored at $-20^{\circ} \mathrm{C}$. Shake flasks with $10 \mathrm{~mL} \mathrm{SM}$ glucose media were inoculated with yeast strains from a cryo-stock and cultivated aerobically at $200 \mathrm{rpm}$ at $30^{\circ} \mathrm{C}$ overnight. The yeast cultures were subsequently diluted to an $\mathrm{OD}_{660}$ of 0.2 in $400 \mathrm{~mL} \mathrm{SM}$ glucose media in $500 \mathrm{~mL}$ shake flasks to gradually reduce the availability of oxygen and incubated overnight. Yeast cultures were transferred to fresh SM media with $40 \mathrm{~g} \mathrm{~L}^{-1}$ glucose and incubated under anaerobic conditions at $30^{\circ} \mathrm{C}$ at $200 \mathrm{rpm}$. After $22 \mathrm{~h}$ of anaerobic incubation $4 \mu \mathrm{g} \mathrm{L}{ }^{-1}$ NBD-cholesterol with $420 \mathrm{mg} \mathrm{L}^{-1}$ Tween 80 were pulsed to the cultures. Samples were taken and washed with PBS $5 \mathrm{~mL} \mathrm{~L}^{-1}$ Tergitol NP-40 pH 7.0 (Sigma-Aldrich) twice before resuspension in PBS and subsequent analysis. Propidium Iodide (PI) (Invitrogen) was added to the sample $(20 \mu \mathrm{M})$ and stained according to the manufacturer's instructions (Boender et al., 2009). PI intercalates with DNA in cells with a compromised cell membrane, which results in red fluorescence. Samples both unstained and stained with PI were analyzed with Accuri C6 flow cytometer (BD Biosciences, Franklin Lakes, NJ) with a $488 \mathrm{~nm}$ laser and fluorescence was measured with emission filter of 533/30 nm (FL1) for NBD-cholesterol and $>670 \mathrm{~nm}$ (FL3) for PI. Cell gating and median fluorescence of cells were determined using FlowJo (v10, BD Bioscience). Cells were gated based on forward side scatter (FSC) and side-scatter (SSC) to exclude potential artifacts or clumping cells. Within this gated population PI positive and negatively stained cells were differentiated based on the cell fluorescence across a FL3 FL1 dimension. Flow cytometric gates were drafted for each yeast species and used for all samples. The gating strategy is given in Supplementary Fig. 8. Fluorescence of a strain was determined by a sample of cells from independent shake-flask cultures and compared to cells from identical unstained cultures of cells with the exact same chronological age. The staining experiment of the strains IMX585, CBS6556 and NBRC1777 samples was repeated twice for reproducibility, the mean and pooled variance was subsequently calculated from the biological duplicates of the two experiments. The NBDC intensity and cell counts obtained from the NBDC experiments are available for re-analysis in Supplementary Data set 1 , and raw flow cytometry plots are depicted in Supplementary Data set 2 .

\subsection{Long read sequencing, assembly, and annotation}

Cells were grown overnight in 500-mL shake flasks containing 100 $\mathrm{mL}$ liquid YPD medium at $30{ }^{\circ} \mathrm{C}$ in an orbital shaker at $200 \mathrm{rpm}$. After 
reaching stationary phase the cells were harvested for a total $\mathrm{OD}_{660}$ of 600 by centrifugation for $5 \mathrm{~min}$ at $4000 \mathrm{~g}$. Genomic DNA of CBS6556 and NBRC1777 was isolated using the Qiagen genomic DNA 100/G kit (Qiagen, Hilden, Germany) according to the manufacturer's instructions. MinION genomic libraries were prepared using the 1D Genomic DNA by ligation (SQK-LSK108) for CBS6556, and the 1D native barcoding Genomic DNA (EXP-NBD103 \& LSK108) for NBRC1777 according to the manufacturer's instructions with the exception of using $80 \%$ EtOH during the 'End Repair/dA-tailing module' step. Flow cell quality was tested by running the MinKNOW platform QC (Oxford Nanopore Technology, Oxford, UK). Flow cells were prepared by removing $20 \mu \mathrm{L}$ buffer and subsequently primed with priming buffer. The DNA library was loaded dropwise into the flow cell for sequencing. The SQK-LSK108 library was sequenced on a R9 chemistry flow cell (FLO-MIN106) for $48 \mathrm{~h}$. Base-calling was performed using Albacore (v2.3.1, Oxford Nanopore Technologies) for CBS6556, and for NBRC1777 with Guppy (v2.1.3, Oxford Nanopore Technologies) using dna_r9.4.1_450bps_flipflop.cfg. CBS6556 reads were assembled using Canu (v1.8) (Koren et al., 2017), and NBRC1777 reads were assembled using Flye (v2.7.1-b1673) (Kolmogorov et al., 2019). Assemblies were polished with Pilon (v1.18) (Walker et al., 2014) using Illumina data available at the Sequence Read Archive under accessions SRX3637961 and SRX3541357. Both de novo genome assemblies were annotated using Funannotate (v1.7.1) (Palmer and Stajich, 2019), trained and refined using de novo transcriptome assemblies (see below), adding functional annotation with Interproscan (v5.25-64.0) (Jones et al., 2014).

\subsection{Illumina sequencing}

Plasmids were sequenced on a MiniSeq (Illumina, San Diego, CA) platform. Library preparation was performed with Nextera XT DNA library preparation according to the manufacturer's instructions (Illumina). The library preparation included the MiniSeq Mid Output kit (300 cycles) and the input \& final DNA was quantified with the Qubit HS dsDNA kit (Life Technologies, Thermo Fisher Scientific). Nucleotide sequences were assembled with SPAdes (Bankevich et al., 2012) and compared to the intended in silico DNA construct. For whole-genome sequencing, yeast cells were harvested from overnight cultures and DNA was isolated with the Qiagen genomic DNA 100/G kit (Qiagen) as described earlier. DNA quantity was measured with the QuBit BR dsDNA kit (Thermo Fisher Scientific). 300 bp paired-end libraries were prepared with the TruSeq DNA PCR-free library prep kit (Illumina) according to the manufacturer's instructions. Short read whole-genome sequencing was performed on a MiSeq platform (Illumina).

\subsection{RNA isolation, sequencing and transcriptome analysis}

Culture broth from chemostat cultures was directly sampled into liquid nitrogen to prevent mRNA turnover. The cell cultures were stored at $-80^{\circ} \mathrm{C}$ and processed within 10 days after sampling. After thawing on ice, cells were harvested by centrifugation. Total RNA was extracted by a 5 min heatshock at $65{ }^{\circ} \mathrm{C}$ with a mix of isoamyl alcohol, phenol and chloroform at a ratio of 125:24:1, respectively (Invitrogen). RNA was extracted from the organic phase with Tris- $\mathrm{HCl}$ and subsequently precipitated by the addition of $3 \mathrm{M} \mathrm{Nac}$-acetate and $40 \%(\mathrm{v} / \mathrm{v})$ ethanol at $-20^{\circ} \mathrm{C}$. Precipitated RNA was washed with ethanol, collected and after drying resuspended in RNAse free water. The quantity of total RNA was determined with a Qubit RNA BR assay kit (Thermo Fisher Scientific). RNA quality was determined by the RNA integrity number with RNA screen tape using a Tapestation (Agilent). RNA libraries were prepared with the TruSeq Stranded mRNA LT protocol (Illumina, \#15031047) and subjected to paired-end sequencing (151 bp read length, NovaSeq Illumina) by Macrogen (Macrogen Europe, Amsterdam, the Netherlands).

Pooled RNAseq libraries were used to perform de novo transcriptome assembly using Trinity (v2.8.3) (Grabherr et al., 2011) which was subsequently used as evidence for both CBS6556 and NBRC1777 genome annotations. RNAseq libraries were mapped into the CBS6556 genome assembly described above, using bowtie (v1.2.1.1) (Langmead et al., 2009) with parameters (-v 0 -k 10 -best -M 1) to allow no mismatches, select the best out of 10 possible alignments per read, and for reads having more than one possible alignment randomly report only one. Alignments were filtered and sorted using samtools (v1.3.1) (Li et al., 2009). Read counts were obtained with featureCounts (v1.6.0) (Liao et al., 2014) using parameters (-B -C) to only count reads for which both pairs are aligned into the same chromosome.

Differential gene expression (DGE) analysis was performed using edgeR (v3.28.1) (McCarthy et al., 2012). Genes with 0 read counts in all conditions were filtered out from the analysis, same as genes with less than 10 counts per million. Counts were normalized using the trimmed mean of M values (TMM) method (Robinson and Oshlack, 2010), and dispersion was estimated using generalized linear models. Differentially expressed genes were then calculated using a log ratio test adjusted with the Benjamini-Hochberg method. Absolute $\log 2$ fold-change values $>2$, false discovery rate $<0.5$, and $\mathrm{P}$ value $<0.05$ were used as significance cutoffs.

Gene set analysis (GSA) based on gene ontology (GO) terms was used to get a functional interpretation of the DGE analysis. For this purpose, GO terms were first obtained for the $S$. cerevisiae CEN.PK113-7D (GCA_002571405.2) and K. marxianus CBS6556 genome annotations using Funannotate and Interproscan as described above. Afterwards, Funannotate compare was used to get (co)ortholog groups of genes generated with ProteinOrtho5 (Lechner et al., 2011) using the following public genome annotations S. cerevisiae S288C (GCF_000146045.2), K. marxianus NBRC1777 (GCA_001417835.1), $K$. marxianus DMKU3-1042 (GCF_001417885.1), in addition to the new genome annotations generated here for $S$. cerevisiae CEN.PK113-7D, and K. marxianus CBS6556 and NBRC1777. Predicted GO terms for S. cerevisiae CEN.PK113-7D and K. marxianus CBS6556 were kept and merged with those from corresponding (co)orthologs from $S$. cerevisiae S288C. Genes with term GO:0005840 (ribosome) were not considered for further analyses. GSA was then performed with Piano (v2.4.0) (Väremo et al., 2013). Gene set statistics were first calculated with the Stouffer, Wilcoxon rank-sum test, and reporter methods implemented in Piano. Afterwards, consensus results were derived by p-value and rank aggregation, considered significant if absolute Fold Change values $>1$. ComplexHeatmap (v2.4.3) (Gu et al., 2016) was used to draw GSA results into Fig. 2, highlighting differentially expressed genes found in a previous study (Tai et al., 2005). DGE and GSA were performed using R (v4.0.2) (R Core Team, 2017).

\subsection{Anaerobic growth experiments}

Anaerobic shake-flask experiments were performed in a Bactron anaerobic workstation (BACTRON300-2, Sheldon Manufacturing, Cornelius, OR) at $30{ }^{\circ} \mathrm{C}$. The gas atmosphere consisted of $85 \% \mathrm{~N}_{2}, 10 \% \mathrm{CO}_{2}$ and $5 \% \mathrm{H}_{2}$ and was maintained anaerobic by a Pd catalyst. The catalyst was re-generated by heating till $160^{\circ} \mathrm{C}$ every week and interchanged by placing it in the airlock whenever the pass-box was used. 50-mL Shake flasks were filled with $40 \mathrm{~mL}$ ( $80 \%$ volumetric) media and placed on an orbital shaker (KS 130 basic, IKA, Staufen, Germany) set at $240 \mathrm{rpm}$ inside the anaerobic chamber. Sterile growth media was placed inside the anaerobic chamber $24 \mathrm{~h}$ prior to inoculation to ensure complete removal of traces of oxygen.

The anaerobic growth ability of the yeast strains was tested on SMGurea with $50 \mathrm{~g} \cdot \mathrm{L}^{-1}$ glucose at $\mathrm{pH} 6.0$ with Tween 80 prepared as described earlier. The growth experiments were started from aerobic pre-cultures on SMG-urea media and the anaerobic shake flasks were inoculated at an $\mathrm{OD}_{660}$ of 0.2 (corresponding to an $\mathrm{OD}_{600}$ of 0.14 ). In order to minimize opening the anaerobic chamber, culture growth was monitored by optical density measurements inside the chamber using an 
Ultrospec 10 cell density meter (Biochrom, Cambridge, UK) at a $600 \mathrm{~nm}$ wavelength. When the optical density of culture no longer increased or decreased new shake-flask cultures were inoculated by serial transfer at an initial $\mathrm{OD}_{600}$ of 0.2 .

\subsection{Laboratory evolution in low oxygen atmosphere}

Adaptive laboratory evolution for strict anaerobic growth was performed in a Bactron anaerobic workstation (BACTRON BAC-X-2E, Sheldon Manufacturing) at $30{ }^{\circ} \mathrm{C}$. 50 -mL Shake flasks were filled with $40 \mathrm{~mL}$ SMG-urea with $50 \mathrm{~g} \mathrm{~L}^{-1}$ glucose and including $420 \mathrm{mg} \mathrm{L}^{-1}$ Tween 80. Subsequently the shake-flask media were inoculated with IMX2323 from glycerol cryo-stock at $\mathrm{OD}_{660}<0.01$ and thereafter placed inside the anaerobic chamber. Due to frequent opening of the pass-box and lack of catalyst inside the pass-box oxygen entry was more permissive. After the optical density of the cultures no longer increased, cultures were transferred to new media by $40-50 x$ serial dilution. For IMS1111, IMS1112, IMS1113 three and for IMS1131, IMS1132, IMS1133 four serial transfers in shake-flask media were performed after which single colony isolates were made by plating on YPD agar media with hygromycin antibiotic at $30{ }^{\circ} \mathrm{C}$ aerobically. Single colony isolates were subsequently restreaked sequentially for three times on the same media before the isolates were propagated in SM glucose media and glycerol cryo stocked.

To determine if an oxygen-limited pre-culture was required for the strict anaerobic growth of IMX2323 strain a cross-validation experiment was performed. In parallel, yeast strains were cultivated in 50-mL shakeflask cultures with SMG-urea with $50 \mathrm{~g} \cdot \mathrm{L}^{-1}$ glucose at $\mathrm{pH} 6.0$ with Tween 80 in both the Bactron anaerobic workstation (BACTRON BAC-X2E, Sheldon Manufacturing) with low levels of oxygen-contamination, and in the Bactron anaerobic workstation (BACTRON300-2, Sheldon Manufacturing) with strict control of oxygen-contamination. After stagnation of growth was observed in the second serial transfer of the shake-flask cultures a $1.5 \mathrm{~mL}$ sample of each culture was taken, sealed, and used to inoculate fresh-media in the other Bactron anaerobic workstation. Simultaneously, the original culture was used to inoculate fresh media in the same Bactron anaerobic workstation, thereby resulting in 4 parallel cultures of each strain of which half were derived from the other Bactron anaerobic workstation.

\subsection{Laboratory evolution in sequential batch reactors}

Laboratory evolution for selection of fast growth at high temperatures was performed in 400-mL MultiFors (Infors Benelux, Velp, the Netherlands) bioreactors with a working volume of $100 \mathrm{~mL}$ for the K. marxianus strain IMS1111 on SMG $20 \mathrm{~g} \mathrm{~L}^{-1}$ glucose media with Tween 80 in triplicate. Anaerobic conditions were created and maintained by continuous aeration of the cultures with $50 \mathrm{~mL} \mathrm{~min}^{-1}(0.5$ vvm) $\mathrm{N}_{2}$ gas and continuous aeration of the media vessels with $\mathrm{N}_{2}$ gas. The $\mathrm{pH}$ was set at 5.0 and maintained by the continuous addition of sterile $2 \mathrm{M} \mathrm{KOH}$. Growth was monitored by analysis of the $\mathrm{CO}_{2}$ in the bioreactor off-gas and a new empty-refill cycle was initiated when the batch time had at least elapsed $15 \mathrm{~h}$ and the $\mathrm{CO}_{2}$ signal dropped to $70 \%$ of the maximum reached in each batch. The dilution factor of each empty-refill cycle was 14.3 -fold ( $100 \mathrm{~mL}$ working volume, $7 \mathrm{~mL}$ residual volume). The first batch fermentation was performed at $30{ }^{\circ} \mathrm{C}$ after which in the second batch the temperature was increased to $42{ }^{\circ} \mathrm{C}$ and maintained at for 18 consecutive sequential batches. After the 18 batch cycle at $42{ }^{\circ} \mathrm{C}$ the culture temperature was again increased to $45^{\circ} \mathrm{C}$ and maintained subsequently. Growth rate was calculated based on the $\mathrm{CO}_{2}$ production as measured by the $\mathrm{CO}_{2}$ fraction in the culture off-gas in essence as described previously (Juergens et al., 2018). In short, the $\mathrm{CO}_{2}$ fraction in the off-gas was converted to a $\mathrm{CO}_{2}$ evolution rate of mmol per hour and subsequently summed over time for each cycle. The corresponding cumulative $\mathrm{CO}_{2}$ profile was transformed to natural log after which the stepwise slope of the log transformed data was calculated.
Subsequently an iterative exclusion of datapoints of the stepwise slope of the $\log$ transformed cumulative $\mathrm{CO}_{2}$ profile was performed with exclusion criteria of more than one standard deviation below the mean.

\subsubsection{Variant calling}

DNA sequencing reads were aligned into the NBRC1777 described above including an additional sequence with TtSTC1 construct, and used to detect sequence variants using a method previously reported (Ortiz-Merino et al., 2018). Briefly, reads were aligned using BWA (v0.7.15-r1142-dirty) (Li and Durbin, 2009), alignments were processed using samtools (v1.3.1) (Li et al., 2009) and Picard tools (v2.20.2-SNAPSHOT) (http://broadinstitute.github.io/picard), and variants were then called using the Genome Analysis Toolkit (v3.8-1-0-gf15c1c3ef) (Auwera et al., 2013) HaplotypeCaller in DISCOVERY and GVCF modes. Variants were only called at sites with minimum variant confidence normalized by unfiltered depth of variant samples (QD) of 20, read depth (DP) $\geq 5$, and genotype quality (GQ) $>$ 20 , excluding a $7.1 \mathrm{~kb}$ region in chromosome 5 containing rDNA. Variants were annotated using the genome annotation described above, including the TtSTC1 construct, with SnpEff (v5.0) (Cingolani et al., 2012) and VCFannotator (http://vcfannotator.sourceforge.net).

\subsection{Statistics}

Statistical test performed are given as two sided with unequal variance $t$-test unless specifically stated otherwise. We denote technical replicates as measurements derived from a single cell culture. Biological replicates are measurements originating from independent cell cultures. Independent experiments are two experiments identical in set-up separated by the difference in execution days. When possible variance from independent experiments with identical setup were pooled together, but independent experiments from time-course experiments (anaerobic growth studies) are reported separately. $p$-values were corrected for multiple-hypothesis testing which is specifically reported each time. No data was excluded based on the resulting data outcome.

\section{Data availability}

Data supporting the findings of this work are available within the paper and source data for all figures in this study are available at the da ta.4TU.nl repository with the https://doi.org/10.4121/13265552.

The raw RNA-sequencing data that supports the findings of this study are available from the Genome Expression Omnibus (GEO) website (http s://www.ncbi.nlm.nih.gov/geo/) with number GSE164344.

Whole-genome sequencing data of the CBS6556, NBRC1777 and evolved strains were deposited at NCBI (https://www.ncbi.nlm.nih. gov/) under BioProject accession number PRJNA679749.

\section{Code availability}

The code that was used to generate the results obtained in this study are archived in a Gitlab repository (https://gitlab.tudelft.nl/rortizmerin o/kmar_anaerobic).

\section{Author's contributions}

WD and JTP designed the study and wrote the manuscript. WD performed molecular cloning, bioreactor cultivation experiment, transcriptome analysis and sterol-uptake experiments. JB contributed to bioreactor cultivation experiments and molecular cloning. FW contributed to the molecular cloning and sterol-uptake experiments. AK and CM contributed to bioreactor experiments and transcriptome studies. PdlT performed plasmid and genome sequencing. RO contributed to transcriptome analysis and performed sequence annotation and assembly. 


\section{Funding}

This work was supported by Advanced Grant (grant \#694633) of the European Research Council to JTP.

\section{Declarations of competing interest}

WD and JTP are co-inventors on a patent application that covers aspects of this work. The authors declare no conflict of interest.

\section{Acknowledgements}

We thank Mark Bisschops and Hannes Jürgens for fruitful discussions. We thank Erik de Hulster for fermentation support and Marcel van den Broek for input on the bioinformatics analyses.

\section{Appendix A. Supplementary data}

Supplementary data to this article can be found online at https://doi. org/10.1016/j.ymben.2021.07.006.

\section{References}

Alimardani, P., Régnacq, M., Moreau-Vauzelle, C., Ferreira, T., Rossignol, T., Blondin, B. Bergès, T., 2004. SUT1-promoted sterol uptake involves the ABC transporter Aus1 and the mannoprotein Dan1 whose synergistic action is sufficient for this process. Biochem. J. 381, 195-202. https://doi.org/10.1042/BJ20040297.

Andreasen, A.A., Stier, T.J.B., 1953a. Anaerobic nutrition of Saccharomyces cerevisiae I. Ergosterol requirement for growth in a defined medium. J. Cell. Physiol. 41, 23-26. https://doi.org/10.1002/jcp.1030410103.

Andreasen, A.A., Stier, T.J.B., 1953b. Anaerobic nutrition of Saccharomyces cerevisiae II. Unsaturated fatty acid requirement for growth in a defined medium. J. Cell. Physiol. 43, 271-281. https://doi.org/10.1002/jcp.1030430303.

Annual World Fuel Ethanol Production, 2020 [WWW Document] Renew. Fuels Assoc. URL https://ethanolrfa.org/statistics/annual-ethanol-production/.

Auwera, G.A., Carneiro, M.O., Hartl, C., Poplin, R., del Angel, G., Levy-Moonshine, A., Jordan, T., Shakir, K., Roazen, D., Thibault, J., Banks, E., Garimella, K.V., Altshuler, D., Gabriel, S., DePristo, M.A., 2013. From FastQ data to high-confidence variant calls: the genome analysis Toolkit best practices pipeline. Curr. Protoc. Bioinforma. 43, 11. https://doi.org/10.1002/0471250953.bi1110s43, 10.111.10.33.

Bakker, B.M., Overkamp, K.M., Van Maris, A.J.A., Kötter, P., Luttik, M.A.H., Van Dijken, J.P., Pronk, J.T., 2001. Stoichiometry and compartmentation of NADH metabolism in Saccharomyces cerevisiae. FEMS Microbiol. Rev. 25, 15-37. https:// doi.org/10.1016/S0168-6445(00)00039-5.

Bankevich, A., Nurk, S., Antipov, D., Gurevich, A.A., Dvorkin, M., Kulikov, A.S., Lesin, V. M., Nikolenko, S.I., Pham, S., Prjibelski, A.D., Pyshkin, A.V., Sirotkin, A.V., Vyahhi, N., Tesler, G., Alekseyev, M.A., Pevzner, P.A., 2012. SPAdes: a new genome assembly algorithm and its applications to single-cell sequencing. J. Comput. Biol. 19, 455-477. https://doi.org/10.1089/cmb.2012.0021.

Black, P.N., DiRusso, C.C., 2007. Yeast acyl-CoA synthetases at the crossroads of fatty acid metabolism and regulation. Biochim. Biophys. Acta Mol. Cell Biol. Lipids 1771, 286-298. https://doi.org/10.1016/j.bbalip.2006.05.003.

Blomqvist, J., Nogue, V.S., Gorwa-Grauslund, M., Passoth, V., 2012. Physiological requirements for growth and competitveness of Dekkera bruxellensis under oxygen limited or anaerobic conditions. Yeast 29, 265-274. https://doi.org/10.1002/yea.

Boender, L.G.M., De Hulster, E.A.F., van Maris, A.J.A., Daran-Lapujade, P.A.S., Pronk, J. T., 2009. Quantitative physiology of Saccharomyces cerevisiae at near-zero specific growth rates. Appl. Environ. Microbiol. 75, 5607-5614. https://doi.org/10.1128/ AEM.00429-09.

Bracher, J.M., de Hulster, E., Koster, C.C., van den Broek, M., Daran, J.M.G., van Maris, A.J.A., Pronk, J.T., 2017. Laboratory evolution of a biotin-requiring Saccharomyces cerevisiae strain for full biotin prototrophy and identification of causal mutations. Appl. Environ. Microbiol. 83, 1-16. https://doi.org/10.1128/ AEM.00892-17.

Choudhary, J., Singh, S., Nain, L., 2016. Thermotolerant fermenting yeasts for simultaneous saccharification fermentation of lignocellulosic biomass. Electron. J. Biotechnol. 21, 82-92. https://doi.org/10.1016/j.ejbt.2016.02.007.

Cingolani, P., Platts, A., Coon, M., Nguyen, T., Wang, L., Land, S.J., Lu, X., Ruden, D.M., 2012. A program for annotating and predicting the effects of single nucleotide polymorphisms, SnpEff: SNPs in the genome of Drosophila melanogaster strain w1118; iso-2; iso-3. Fly 6, 80-92.

da Costa, B.L.V., Basso, T.O., Raghavendran, V., Gombert, A.K., 2018. Anaerobiosis revisited: growth of Saccharomyces cerevisiae under extremely low oxygen availability. Appl. Microbiol. Biotechnol. 102, 2101-2116. https://doi.org/10.1007/ s00253-017-8732-4

Daran-Lapujade, P., Daran, J.-M., van Maris, A.J.A., de Winde, J.H., Pronk, J.T., 2008. Chemostat-based micro-array analysis in baker's yeast. In: Advances in Microbial Physiology, pp. 257-417. https://doi.org/10.1016/S0065-2911(08)00004-0.
Dashko, S., Zhou, N., Compagno, C., Piškur, J., 2014. Why, when, and how did yeast evolve alcoholic fermentation? FEMS Yeast Res. 14, 826-832. https://doi.org/ 10.1111/1567-1364.12161.

Dekker, W.J.C., Wiersma, S.J., Bouwknegt, J., Mooiman, C., Pronk, J.T., 2019. Anaerobic growth of Saccharomyces cerevisiae CEN.PK113-7D does not depend on synthesis or supplementation of unsaturated fatty acids. FEMS Yeast Res. 19 https://doi.org/ 10.1093/femsyr/foz060.

Entian, K.-D., Kötter, P., 2007. 25 yeast genetic strain and plasmid collections. In: Methods in Microbiology, pp. 629-666. https://doi.org/10.1016/S0580-9517(06) 36025-4.

Favaro, L., Jansen, T., van Zyl, W.H., 2019. Exploring industrial and natural Saccharomyces cerevisiae strains for the bio-based economy from biomass: the case of bioethanol. Crit. Rev. Biotechnol. 39, 800-816. https://doi.org/10.1080/ 07388551.2019.1619157.

Fonseca, G.G., Heinzle, E., Wittmann, C., Gombert, A.K., 2008. The yeast Kluyveromyces marxianus and its biotechnological potential. Appl. Microbiol. Biotechnol. 79, 339-354. https://doi.org/10.1007/s00253-008-1458-6.

Gietz, R.D., Woods, R.A., 2001. Genetic transformation of yeast. Biotechniques 30, 816-831. https://doi.org/10.2144/01304rv02.

Gojković, Z., Knecht, W., Zameitat, E., Warneboldt, J., Coutelis, J.B., Pynyaha, Y., Neuveglise, C., Møller, K., Löffler, M., Piškur, J., 2004. Horizontal gene transfer promoted evolution of the ability to propagate under anaerobic conditions in yeasts. Mol. Genet. Genom. 271, 387-393. https://doi.org/10.1007/s00438-004-0995-7.

Grabherr, M.G., Haas, B.J., Yassour, M., Levin, J.Z., Thompson, D.A., Amit, I., Adiconis, X., Fan, L., Raychowdhury, R., Zeng, Q., Chen, Z., Mauceli, E., Hacohen, N., Gnirke, A., Rhind, N., Di Palma, F., Birren, B.W., Nusbaum, C., Lindblad-Toh, K., Friedman, N., Regev, A., 2011. Full-length transcriptome assembly from RNA-Seq data without a reference genome. Nat. Biotechnol. 29, 644-652. https://doi.org/10.1038/nbt.1883.

Gu, Z., Eils, R., Schlesner, M., 2016. Complex heatmaps reveal patterns and correlations in multidimensional genomic data. Bioinformatics 32, 2847-2849. https://doi.org/ 10.1093/bioinformatics/btw313.

Guo, L., Pang, Z., Gao, C., Chen, X., Liu, L., 2020. Engineering microbial cell morphology and membrane homeostasis toward industrial applications. Curr. Opin. Biotechnol. 66, 18-26. https://doi.org/10.1016/j.copbio.2020.05.004.

Hassing, E.J., de Groot, P.A., Marquenie, V.R., Pronk, J.T., Daran, J.M.G., 2019. Connecting central carbon and aromatic amino acid metabolisms to improve de novo 2-phenylethanol production in Saccharomyces cerevisiae. Metab. Eng. 56, 165-180. https://doi.org/10.1016/j.ymben.2019.09.011.

Hong, J., Wang, Y., Kumagai, H., Tamaki, H., 2007. Construction of thermotolerant yeast expressing thermostable cellulase genes. J. Biotechnol. 130, 114-123. https://doi. org/10.1016/j.jbiotec.2007.03.008.

Hughes, S.R., Bang, S.S., Cox, E.J., Schoepke, A., Ochwat, K., Pinkelman, R., Nelson, D., Qureshi, N., Gibbons, W.R., Kurtzman, C.P., Bischoff, K.M., Liu, S., Cote, G.L., Rich, J.O., Jones, M.a., Cedeño, D., Doran-Peterson, J., Riaño-Herrera, N.M., Rodríguez-Valencia, N., López-Núñez, J.C., 2013. Automated UV-C mutagenesis of Kluyveromyces marxianus NRRL Y-1109 and selection for microaerophilic growth and ethanol production at elevated temperature on biomass sugars. J. Lab. Autom. 18, 276-290. https://doi.org/10.1177/2211068213480037.

Jacquier, N., Schneiter, R., 2010. Ypk1, the yeast orthologue of the human serum- and glucocorticoid-induced kinase, is required for efficient uptake of fatty acids. J. Cell Sci. 123, 2218-2227. https://doi.org/10.1242/jcs.063073.

Jansen, M.L.A., Bracher, J.M., Papapetridis, I., Verhoeven, M.D., de Bruijn, H., de Waal, P.P., van Maris, A.J.A., Klaassen, P., Pronk, J.T., 2017. Saccharomyces cerevisiae strains for second-generation ethanol production: from academic exploration to industrial implementation. FEMS Yeast Res. 17, 1-20. https://doi. org/10.1093/femsyr/fox044.

Jeong, H., Lee, D.H., Kim, S.H., Kim, H.J., Lee, K., Song, J.Y., Kim, B.K., Sung, B.H., Park, J.C., Sohn, J.H., Koo, H.M., Kim, J.F., 2012. Genome sequence of the thermotolerant yeast Kluyveromyces marxianus var. marxianus KCTC 17555. Eukaryot. Cell 11, 1584-1585. https://doi.org/10.1128/EC.00260-12.

Jones, P., Binns, D., Chang, H.Y., Fraser, M., Li, W., McAnulla, C., McWilliam, H. Maslen, J., Mitchell, A., Nuka, G., Pesseat, S., Quinn, A.F., Sangrador-Vegas, A., Scheremetjew, M., Yong, S.Y., Lopez, R., Hunter, S., 2014. InterProScan 5: genomescale protein function classification. Bioinformatics 30, 1236-1240. https://doi.org/ 10.1093/bioinformatics/btu031.

Jordá, T., Puig, S., 2020. Regulation of ergosterol biosynthesis in Saccharomyces cerevisiae. Genes 11, 795. https://doi.org/10.3390/genes11070795.

Juergens, H., Niemeijer, M., Jennings-Antipov, L.D., Mans, R., Morel, J., van Maris, A.J. A., Pronk, J.T., Gardner, T.S., 2018. Evaluation of a novel cloud-based software platform for structured experiment design and linked data analytics. Sci. Data 5, 1-12. https://doi.org/10.1038/sdata.2018.195.

Kiers, J., Zeeman, A.-M., Luttik, M., Thiele, C., Castrillo, J.I., Steensma, H.Y., Dijken, J.P. Van, Pronk, J.T., 1998. Regulation of alcoholic fermentation in batch and chemostat cultures of Kluyveromyces lactis CBS 2359. Yeast 14, 459-469. https://doi.org/ 10.1002/(SICI)1097-0061(19980330)14.

Kolmogorov, M., Yuan, J., Lin, Y., Pevzner, P.A., 2019. Assembly of long, error-prone reads using repeat graphs. Nat. Biotechnol. 37, 540-546. https://doi.org/10.1038/ s41587-019-0072-8.

Koren, S., Walenz, B.P., Berlin, K., Miller, J.R., Bergman, N.H., Phillippy, A.M., 2017. Canu: scalable and accurate long-read assembly via adaptive $\kappa$-mer weighting and repeat separation. Genome Res. 27, 722-736. https://doi.org/10.1101/ gr.215087.116.

Laman Trip, D.S., Youk, H., 2020. Yeasts collectively extend the limits of habitable temperatures by secreting glutathione. Nat. Microbiol. 5, 943-954. https://doi.org/ 10.1038/s41564-020-0704-2. 
Landry, B.D., Doyle, J.P., Toczyski, D.P., Benanti, J.A., 2012. F-box protein specificity for G1 cyclins is dictated by subcellular localization. PLoS Genet. 8 https://doi.org/ 10.1371/journal.pgen.1002851 e1002851.

Langmead, B., Trapnell, C., Pop, M., Salzberg, S.L., 2009. Ultrafast and memory-efficient alignment of short DNA sequences to the human genome. Genome Biol. 10 https:// doi.org/10.1186/gb-2009-10-3-r25. R25.

Lechner, M., Findeiß, S., Steiner, L., Marz, M., Stadler, P.F., Prohaska, S.J., 2011. Proteinortho: detection of (Co-)orthologs in large-scale analysis. BMC Bioinf. 12 , 124. https://doi.org/10.1186/1471-2105-12-124.

Lee, M.E., DeLoache, W.C., Cervantes, B., Dueber, J.E., 2015. A highly characterized yeast Toolkit for modular, multipart assembly. ACS Synth. Biol. 4, 975-986. https:// doi.org/10.1021/sb500366v.

Li, H., Durbin, R., 2009. Fast and accurate short read alignment with Burrows-Wheeler transform. Bioinformatics 25, 1754-1760. https://doi.org/10.1093/bioinformatics/ btp324.

Li, H., Handsaker, B., Wysoker, A., Fennell, T., Ruan, J., Homer, N., Marth, G., Abecasis, G., Durbin, R., 2009. The sequence alignment/map format and SAMtools. Bioinformatics 25, 2078-2079. https://doi.org/10.1093/bioinformatics/btp352.

Liao, Y., Smyth, G.K., Shi, W., 2014. FeatureCounts: an efficient general purpose program for assigning sequence reads to genomic features. Bioinformatics 30, 923-930. https://doi.org/10.1093/bioinformatics/btt656.

Lorenz, R., Bernhart, S.H., Höner zu Siederdissen, C., Tafer, H., Flamm, C., Stadler, P.F., Hofacker, I.L., 2011. ViennaRNA package 2.0. Algorithm Mol. Biol. 6, 26. https:// doi.org/10.1186/1748-7188-6-26.

Madeira, J.V., Gombert, A.K., 2018. Towards high-temperature fuel ethanol production using Kluyveromyces marxianus: on the search for plug-in strains for the Brazilian sugarcane-based biorefinery. Biomass Bioenergy 119, 217-228. https://doi.org/ 10.1016/j.biombioe.2018.09.010.

Mans, R., van Rossum, H.M., Wijsman, M., Backx, A., Kuijpers, N.G.A., van den Broek, M., Daran-Lapujade, P., Pronk, J.T., van Maris, A.J.A., Daran, J.M.G., 2015. CRISPR/Cas9: a molecular Swiss army knife for simultaneous introduction of multiple genetic modifications in Saccharomyces cerevisiae. FEMS Yeast Res. 15, 1-15. https://doi.org/10.1093/femsyr/fov004.

Marek, M., Milles, S., Schreiber, G., Daleke, D.L., Dittmar, G., Herrmann, A., Müller, P., Pomorski, T.G., 2011. The yeast plasma membrane ATP binding cassette (ABC) transporter Aus1: purification, characterization, and the effect of lipids on its activity. J. Biol. Chem. 286, 21835-21843. https://doi.org/10.1074/jbc. M111.244525.

Marek, M., Silvestro, D., Fredslund, M.D., Andersen, T.G., Pomorski, T.G., 2014. Serum albumin promotes ATP-binding cassette transporter-dependent sterol uptake in yeast. FEMS Yeast Res. 14, 1223-1233. https://doi.org/10.1111/1567-1364.12219.

Mashego, M.R., van Gulik, W.M., Vinke, J.L., Heijnen, J.J., 2003. Critical evaluation of sampling techniques for residual glucose determination in carbon-limited chemostat culture of Saccharomyces cerevisiae. Biotechnol. Bioeng. 83, 395-399. https://doi org/10.1002/bit.10683.

McCarthy, D.J., Chen, Y., Smyth, G.K., 2012. Differential expression analysis of multifactor RNA-Seq experiments with respect to biological variation. Nucleic Acids Res. 40, 4288-4297. https://doi.org/10.1093/nar/gks042.

Mejía-Barajas, J.A., Alvarez-Navarrete, M., Saavedra-Molina, A., Campos-García, J., Valenzuela-Vázquez, U., Amaya-Delgado, L., Arellano-Plaza, M., 2018. SecondGeneration bioethanol production through a simultaneous saccharificationfermentation process using Kluyveromyces marxianus thermotolerant yeast. In: Special Topics in Renewable Energy Systems. InTech. https://doi.org/10.5772/ intechopen.78052.

Merico, A., Sulo, P., Piškur, J., Compagno, C., 2007. Fermentative lifestyle in yeasts belonging to the Saccharomyces complex. FEBS J. 274, 976-989. https://doi.org/ 10.1111/j.1742-4658.2007.05645.x.

Mooiman, C., Bouwknegt, J., Dekker, W.J.C., Wiersma, S.J., Ortiz-Merino, R.A., de Hulster, E., Pronk, J.T., 2021. Critical parameters and procedures for anaerobic cultivation of yeasts in bioreactors and anaerobic chambers. FEMS Yeast Res. 21, 1-13. https://doi.org/10.1093/femsyr/foab035.

Nagy, M., Lacroute, F., Thomas, D., 1992. Divergent evolution of pyrimidine biosynthesis between anaerobic and aerobic yeasts. Proc. Natl. Acad. Sci. U. S. A 89, 8966-8970. https://doi.org/10.1073/pnas.89.19.8966.

Nijkamp, J.F., van den Broek, M., Datema, E., de Kok, S., Bosman, L., Luttik, M. a, DaranLapujade, P., Vongsangnak, W., Nielsen, J., Heijne, W.H., Klaassen, P., Paddon, C.J., Platt, D., Kotter, P., van Ham, R.C., Reinders, M.J., Pronk, J.T., Ridder, D. De, Daran, J.-M., 2012. De novo sequencing, assembly and analysis of the genome of the laboratory strain Saccharomyces cerevisiae CEN.PK113-7D, a model for modern industrial biotechnology. Microb. Cell Factories 11, 36. https://doi.org/10.1186/ 1475-2859-11-36.

Ortiz-Merino, R.A., Varela, J.A., Coughlan, A.Y., Hoshida, H., da Silveira, W.B., Wilde, C., Kuijpers, N.G.A., Geertman, J.-M., Wolfe, K.H., Morrissey, J.P., 2018. Ploidy variation in Kluyveromyces marxianus separates dairy and non-dairy isolates. Front. Genet. 9, 1-16. https://doi.org/10.3389/fgene.2018.00094.

Palmer, J., Stajich, J., 2019. Funannotate. https://doi.org/10.5281/zenodo.3548120.

Papapetridis, I., Goudriaan, M., Vázquez Vitali, M., De Keijzer, N.A., Van Den Broek, M., Van Maris, A.J.A., Pronk, J.T., 2018. Optimizing anaerobic growth rate and fermentation kinetics in Saccharomyces cerevisiae strains expressing Calvin-cycle enzymes for improved ethanol yield. Biotechnol. Biofuels 11, 1-17. https://doi.org/ 10.1186/s13068-017-1001-z.

Passi, S., Picardo, M., De Luca, C., Nazzaro-Porro, M., Rossi, L., Rotilio, G., 1993. Saturated dicarboxylic acids as products of unsaturated fatty acid oxidation. Biochim. Biophys. Acta Lipids Lipid. Metabol. 1168, 190-198. https://doi.org/ 10.1016/0005-2760(93)90124-R
Perli, T., Wronska, A.K., Ortiz-Merino, R.A., Pronk, J.T., Daran, J.M., 2020. Vitamin requirements and biosynthesis in Saccharomyces cerevisiae. Yeast 1-22. https://doi. org/10.1002/yea.3461.

Postma, E., Verduyn, C., Scheffers, W.A., Van Dijken, J.P., 1989. Enzymic analysis of the crabtree effect in glucose-limited chemostat cultures of Saccharomyces cerevisiae. Appl. Environ. Microbiol. 55, 468-477. https://doi.org/10.1128/aem.55.2.468477.1989.

R Core Team, 2017. R: A Language and Environment for Statistical Computing.

Rajkumar, A.S., Varela, J.A., Juergens, H., Daran, J.G., Morrissey, J.P., 2019. Biological parts for Kluyveromyces marxianus synthetic biology. Front. Bioeng. Biotechnol. 7, 1-15. https://doi.org/10.3389/fbioe.2019.00097.

Riley, R., Haridas, S., Wolfe, K.H., Lopes, M.R., Hittinger, C.T., Göker, M., Salamov, A.A., Wisecaver, J.H., Long, T.M., Calvey, C.H., Aerts, A.L., Barry, K.W., Choi, C., Clum, A., Coughlan, A.Y., Deshpande, S., Douglass, A.P., Hanson, S.J., Klenk, H.-P., LaButti, K.M., Lapidus, A., Lindquist, E.A., Lipzen, A.M., Meier-Kolthoff, J.P., Ohm, R.A., Otillar, R.P., Pangilinan, J.L., Peng, Y., Rokas, A., Rosa, C.A., Scheuner, C., Sibirny, A.A., Slot, J.C., Stielow, J.B., Sun, H., Kurtzman, C.P., Blackwell, M., Grigoriev, I.V., Jeffries, T.W., 2016. Comparative genomics of biotechnologically important yeasts. Proc. Natl. Acad. Sci. U. S. A 113, 9882-9887. https://doi.org/10.1073/pnas.1603941113.

Robinson, M.D., Oshlack, A., 2010. A scaling normalization method for differential expression analysis of RNA-seq data. Genome Biol. 11 https://doi.org/10.1186/gb2010-11-3-r25.

Rouwenhorst, R.J., Visser, L.E., Van Der Baan, A.A., Scheffers, W.A., Van Dijken, J.P., 1988. Production, distribution, and kinetic properties of inulinase in continuous cultures of Kluyveromyces marxianus CBS 6556. Appl. Environ. Microbiol. 54, 1131-1137. https://doi.org/10.1128/aem.54.5.1131-1137.1988.

Schüller, H.-J., 2003. Transcriptional control of nonfermentative metabolism in the yeast Saccharomyces cerevisiae. Curr. Genet. 43, 139-160. https://doi.org/10.1007/ s00294-003-0381-8.

Seret, M.L., Diffels, J.F., Goffeau, A., Baret, P.V., 2009. Combined phylogeny and neighborhood analysis of the evolution of the ABC transporters conferring multiple drug resistance in hemiascomycete yeasts. BMC Genom. 10, 459. https://doi.org/ 10.1186/1471-2164-10-459.

Shi, N.Q., Jeffries, T.W., 1998. Anaerobic growth and improved fermentation of Pichia stipitis bearing a URA1 gene from Saccharomyces cerevisiae. Appl. Microbiol. Biotechnol. 50, 339-345. https://doi.org/10.1007/s002530051301.

Snoek, I.S.I., Steensma, H.Y., 2007. Factors involved in anaerobic growth of Saccharomyces cerevisiae. Yeast 24, 1-10. https://doi.org/10.1002/yea.

Snoek, I.S.I., Steensma, H.Y., 2006. Why does Kluyveromyces lactis not grow under anaerobic conditions? Comparison of essential anaerobic genes of Saccharomyces cerevisiae with the Kluyveromyces lactis genome. FEMS Yeast Res. 6, 393-403. https://doi.org/10.1111/j.1567-1364.2005.00007.x.

Solis-Escalante, D., Kuijpers, N.G. a, Bongaerts, N., Bolat, I., Bosman, L., Pronk, J.T., Daran, J.M., Daran-Lapujade, P., 2013. amdSYM, A new dominant recyclable marker cassette for Saccharomyces cerevisiae. FEMS Yeast Res. 13, 126-139. https://doi.org/ 10.1111/1567-1364.12024.

Stovicek, V., Holkenbrink, C., Borodina, I., 2017. CRISPR/Cas system for yeast genome engineering: advances and applications. FEMS Yeast Res. 17, 1-16. https://doi.org/ 10.1093/femsyr/fox030.

Tai, S.L., Boer, V.M., Daran-Lapujade, P., Walsh, M.C., De Winde, J.H., Daran, J.M., Pronk, J.T., 2005. Two-dimensional transcriptome analysis in chemostat cultures: combinatorial effects of oxygen availability and macronutrient limitation in Saccharomyces cerevisiae. J. Biol. Chem. 280, 437-447. https://doi.org/10.1074/jbc. M410573200.

Takishita, K., Chikaraishi, Y., Leger, M.M., Kim, E., Yabuki, A., Ohkouchi, N., Roger, A.J., 2012. Lateral transfer of tetrahymanol-synthesizing genes has allowed multiple diverse eukaryote lineages to independently adapt to environments without oxygen. Biol. Direct 7, 5. https://doi.org/10.1186/1745-6150-7-5.

Tetsuya, G., Tsuji, M., Inoue, H., Yano, S., Hoshino, T., Matsushika, A., 2013. Bioethanol production from lignocellulosic biomass by a novel Kluyveromyces marxianus strain. Biosci. Biotechnol. Biochem. 77, 1505-1510. https://doi.org/10.1271/bbb.130173.

Thorwall, S., Schwartz, C., Chartron, J.W., Wheeldon, I., 2020. Stress-tolerant nonconventional microbes enable next-generation chemical biosynthesis. Nat. Chem. Biol. 16, 113-121. https://doi.org/10.1038/s41589-019-0452-x.

Van Eunen, K., Bouwman, J., Daran-Lapujade, P., Postmus, J., Canelas, A.B., Mensonides, F.I.C., Orij, R., Tuzun, I., Van Den Brink, J., Smits, G.J., Van Gulik, W. M., Brul, S., Heijnen, J.J., De Winde, J.H., Teixeira De Mattos, M.J., Kettner, C., Nielsen, J., Westerhoff, H.V., Bakker, B.M., 2010. Measuring enzyme activities under standardized in vivo-like conditions for systems biology. FEBS J. 277, 749-760. https://doi.org/10.1111/j.1742-4658.2009.07524.x.

van Urk, H., Postma, E., Scheffers, W.A., van Dijken, J.P., 1989. Glucose transport in crabtree-positive and crabtree-negative yeasts. J. Gen. Microbiol. 135, 2399-2406.

Väremo, L., Nielsen, J., Nookaew, I., 2013. Enriching the gene set analysis of genomewide data by incorporating directionality of gene expression and combining statistical hypotheses and methods. Nucleic Acids Res. 41, 4378-4391. https://doi. org/10.1093/nar/gkt111.

Verduyn, C., Postma, E., Scheffers, W.A., van Dijken, J.P., 1990. Physiology of Saccharomyces cerevisiae in anaerobic glucose-limited chemostat cultures. J. Gen. Microbiol. 136, 395-403. https://doi.org/10.1099/00221287-136-3-395.

Visser, W., Scheffers, W.A., Batenburg-Van der Vegte, W.H., Van Dijken, J.P., 1990. Oxygen requirements of yeasts. Appl. Environ. Microbiol. 56, 3785-3792.

von Meyenburg, K., 1969. Katabolit-Repression und der Sprossungszyklus von Saccharomyces cerevisiae. ETH Zürich. https://doi.org/10.3929/ethz-a-000099923.

Walker, B.J., Abeel, T., Shea, T., Priest, M., Abouelliel, A., Sakthikumar, S., Cuomo, C. a, Zeng, Q., Wortman, J., Young, S.K., Earl, A.M., 2014. Pilon : an integrated tool for 
comprehensive microbial variant detection and genome assembly improvement. PloS One 9. https://doi.org/10.1371/journal.pone.0112963.

Weusthuis, R.A., Lamot, I., van der Oost, J., Sanders, J.P.M., 2011. Microbial production of bulk chemicals: development of anaerobic processes. Trends Biotechnol. 29, 153-158. https://doi.org/10.1016/j.tibtech.2010.12.007.

Weusthuis, R.A., Visser, W., Pronk, J.T., Scheffers, W.A., Van Dijken, J.P., 1994. Effects of oxygen limitation on sugar metabolism in yeasts: a continuous-culture study of the Kluyver effect. Microbiology 140, 703-715. https://doi.org/10.1099/00221287140-4-703.

Wiersma, S.J., Mooiman, C., Giera, M., Pronk, J.T., 2020. Squalene-tetrahymanol cyclase expression enables sterol-independent growth of Saccharomyces cerevisiae. Appl. Environ. Microbiol. 86, 1-15. https://doi.org/10.1128/AEM.00672-20.
Wilcox, L.J., Balderes, D.A., Wharton, B., Tinkelenberg, A.H., Rao, G., Sturley, S.L., 2002. Transcriptional profiling identifies two members of the ATP-binding cassette transporter superfamily required for sterol uptake in yeast. J. Biol. Chem. 277, 32466-32472. https://doi.org/10.1074/jbc.M204707200.

Wilkins, M.R., Mueller, M., Eichling, S., Banat, I.M., 2008. Fermentation of xylose by the thermotolerant yeast strains Kluyveromyces marxianus IMB2, IMB4, and IMB5 under anaerobic conditions. Process Biochem. 43, 346-350. https://doi.org/10.1016/j. procbio.2007.12.011.

Zavrel, M., Hoot, S.J., White, T.C., 2013. Comparison of sterol import under aerobic and anaerobic conditions in three fungal species, Candida albicans, Candida glabrata, and Saccharomyces cerevisiae. Eukaryot. Cell 12, 725-738. https://doi.org/10.1128/ EC.00345-12. 